\title{
The young Australian loneliness survey Understanding loneliness in adolescence and young adulthood
}

A report prepared for the Victorian Health Promotion Foundation

Dr Michelle H Lim

Dr Robert Eres

Ms Claire Peck

The Iverson Health Innovation Research Institute, and Centre for Mental Health, Swinburne University of Technology. 
The young Australian loneliness survey

Dr Michelle H. Lim, Dr Robert Eres, and Ms Claire Peck are at the Iverson Health Innovation Research Institute, and Centre for Mental Health, Swinburne University of Technology.

Corresponding author: Dr Michelle H Lim,

Iverson Health Innovation Research Institute \& Centre for Mental Health,

Swinburne University of Technology.

Hawthorn, 3122.

Phone: +61392145109

Email: $\underline{\text { mlim@swin.edu.au }}$

The authors declare no conflict of interests. 


\section{Executive summary}

Loneliness is defined as a subjective feeling of social isolation that is more related to the perceived quality rather than the quantity of relationships ${ }^{[1-3]}$. Loneliness has been associated with poorer health outcomes ${ }^{[4-6]}$ and has been identified as an emerging public health problem ${ }^{[7]}$. Loneliness affects everyone ${ }^{[8]}$ and young people are particularly vulnerable ${ }^{[9]}$. However, the prevalence and impact of loneliness on young Australians is unclear.

The Young Australian Loneliness Survey commissioned by VicHealth is the first known study to examine loneliness severity in a large cohort of young Victorians aged 12 to 25 years. This report is in line with the new VicHealth Mental Wellbeing Strategy 2019-2023 on promoting social connections among young people.

This research was jointly conducted with the Social Health and Wellbeing Laboratory, at the Iverson Health Innovation Institute, at Swinburne University of Technology. The main study aim was to examine the prevalence of loneliness and social isolation in adolescents (12-17 years old) and young adults (18-25 years old) residing in Victoria, Australia. We also examine the influence of known factors on loneliness, and this includes mental health factors such as social anxiety and depression, emotion regulation, and affect.

\section{Research summary}

A total of 1,520 participants completed an online survey. There were 650 adolescent participants aged 12 to 17 years old and 870 young adults aged 18 to 25 years old. Demographic factors, loneliness, social isolation risk, known mental health correlates such as social anxiety and depression, emotion regulation, and positive and negative affect measures were collected. 


\section{Key findings}

1. More than one in four young Victorians reported problematic ${ }^{1}$ levels of loneliness, specifically, one in six adolescents and one in three young adults. Overall, adolescents reported significantly lower levels of loneliness than young adults.

2. Almost one in three young Victorians reported themselves to be of high social isolation risk which was measured via frequency of contact with family and friends. Overall, adolescents also reported less social isolation risk than young adults.

3. Those who are lonelier are at an increased risk of poorer mental health outcomes. Loneliness is associated with an increased likelihood of experiencing higher depression and social anxiety.

4. Adolescents compared with young adults consistently performed better on various factors. Adolescents reported lower depression, social anxiety, negative affect, and more positive affect when compared with young adults.

5. Overall, social isolation risk, mental health symptom severity, affect, and emotion regulation all significantly predicted loneliness across the entire sample.

\section{Future directions}

These findings provide preliminary evidence on the loneliness severity in Victorian adolescents and young people. Young adults were significantly lonelier than adolescents in this study. Furthermore, social isolation risk may play a role in contributing to loneliness although this relationship is modest. Targeting loneliness may also alleviate more problematic mental health outcomes such as depression and social anxiety within preventative mental health and wellbeing programs. Other factors such as the ability to experience positive affect and demonstrate adaptive emotion regulation strategies such as cognitive reappraisal, may further enhance one's ability to develop meaningful relationships with others and inadvertently reduce loneliness. It is possible that early intervention programs that focus on promoting good social health before 18 years of age may also protect young people from developing more problematic levels of loneliness in early adulthood.

Please note that the following report is unpublished and prepared in accordance to VicHealth's recommendations. A version of these data is expected to be submitted for publication elsewhere.

\footnotetext{
${ }^{1}$ We defined problematic levels of loneliness when participants scored above 52 on a well-known psychometrically validated loneliness scale. This cut off score is also consistent with the 2018 Australian Loneliness Report ${ }^{[7]}$.
} 


\section{Abstract}

Purpose: Loneliness is well known to be associated with poorer health outcomes and has been identified as an emerging public health problem. While loneliness affects people of all ages, adolescents and young adults are thought to be particularly vulnerable. This is unusual given that these age groups are often perceived to be well-integrated into strong social structures such as schools. The main study aim was to examine the prevalence of loneliness in adolescents and young adults, residing in Victoria, Australia.

Method: A total of 1,520 participants completed an online survey. Of this, there were 650 adolescent participants aged 12 to 17 years old $(M=13.75, S D=1.63)$ and 870 young adults aged 18 to 25 years old $(M=21$. 38, $S D=2.32)$. Demographic factors, loneliness, social isolation risk, known mental health correlates such as social anxiety and depression, emotion regulation, and positive and negative affect measures were collected.

Results: Around one in four young Victorians reported problematic levels of loneliness. Overall, adolescents reported significantly less loneliness when compared with young adults. Adolescents (27.26\%) also reported less social isolation risk than young adults (35.77\%). Adolescents reported better outcomes overall, when compared with young adults: this includes lower depression, social anxiety, negative affect and more positive affect. However, social isolation risk, mental health symptom severity, affect, and emotion regulation all significantly predicted loneliness across the sample.

Discussion: Young adults were significantly lonelier than adolescents in this study. Although the relationship between social isolation and loneliness is modest, having fewer contacts with friends and family predicts loneliness. Other factors that predict loneliness include mental health symptoms such as social anxiety and depression, affect and emotion regulation. Although addressing loneliness in adolescents and young people is likely to yield more positive health outcomes, there is a lack of evidence-based programs mitigating loneliness as a primary target. Programs that can teach individuals to reappraise social situations and strategies to signal a willingness to connect may be beneficial. These programs are likely to be more effective if they are also augmented by the provision of social opportunities. 


\section{The Young Australian Loneliness Survey: understanding loneliness in adolescence and young adulthood}

\section{Definition}

Loneliness is defined as a set of aversive feelings that arise when there is a discrepancy between desired and actual social relationships ${ }^{[2]}$. It is an emotional state that is characterized by subjective perceptions of social isolation and can be seen as a marker that one's relationships may be inadequate or failing to meet expectations ${ }^{[3,10]}$.

Loneliness may be alleviated through increased social interactions. Individuals who are not embedded within a rich social environment are at increased risk of being trapped in the perpetuating cycle of loneliness ${ }^{[1]}$. Unfortunately, increasing the number of social opportunities is not enough to reduce loneliness ${ }^{[11]}$. This is because loneliness is not the same as being alone or socially isolated and is not even strongly correlated with time spent alone ${ }^{[4]}$. While social isolation and loneliness are somewhat related, these constructs can also be conceptualised as being independent and indeed can vary independently. For example, one can be socially isolated but not lonely, or one can be socially connected but lonely.

Research has indicated that even those who are well connected within strong social structures (e.g., school, university, sporting and interest groups) may continue to report problematic loneliness (i.e., higher levels of loneliness). Structural social relationship indicators such as the degree of integration within social networks (including contact with others and participation in social activities) has been showed to be poorly associated with loneliness $\left(r=.20-.30 ;{ }^{[12,13]}\right)$. Adolescents and young adults are known to be socially integrated within educational institutions, recreational activities, work, and social media ${ }^{[14-16]}$, but have been identified as a group who is at risk of experiencing problematic loneliness. In children (aged 11 years), the frequency of school involvement is also only moderately correlated $(r=-.40, p<.01)^{[17]}$. For young adults (assessed at 18 years of age), social isolation (specifically access to supportive relationships) and loneliness have been moderately correlated ( $r=$ $0.39)^{[18]}$.

\section{Health impacts of loneliness}

Problematic levels of loneliness is well known to be associated with poorer health outcomes from poorer mental health ${ }^{[19,20]}$, higher inflammatory responses (i.e., bodily responses to disease and injury) ${ }^{[21]}$, and poorer cardiovascular health ${ }^{[4]}$. Loneliness also contributes to higher mortality rates. 
Holt-Lunstad and colleagues (2015) found in their meta-analytic review that the likelihood of early mortality is equivalent for people who are socially isolated (odds ratio $=1.29 ; 95 \% ; \mathrm{Cl} 1.06,1.56$ ), living alone (odds ratio $=1.32 ; 95 \% ; \mathrm{Cl} 1.14,1.53$ ), or lonely (odds ratio $=1.26,95 \% ; \mathrm{Cl} 1.04,1.53)^{[22]}$.

Unfortunately, adolescence and young adulthood is also associated with multiple social stressors from bullying ${ }^{[23,24]}$, emerging mental health issues ${ }^{[25]}$ and social vulnerability ${ }^{[26]}$. In a UK sample, loneliness in early childhood and adolescence (assessed at age 7) led to higher depression, poorer general health, more frequent doctor visits, and increased alcohol consumption (assessed at age 17) ${ }^{[27]}$. In longitudinal American epidemiological studies, researchers found that adolescent loneliness (those aged 11 to 20) was associated with higher incidence of diagnosed depression, poorer self-rated health, and more metabolic risk factors to cardiovascular disease ${ }^{[28]}$. While parental support can offset these health consequences, adolescents who are lonely still remain at a higher risk of poor health outcomes in adulthood ${ }^{[28]}$.

\section{Prevalence rates across age cohorts}

A recent review of loneliness across the lifespan indicated that the rate of loneliness can range from $11-20 \%$ for adolescents (12-15 years old) to $20-71 \%$ for young adults (15-21 years), significantly more than other age groups ${ }^{[8]}$. However, there is variability in the prevalence rate across studies ${ }^{[29]}$ and this may relate to how many researchers often employ a single-item approach to measure loneliness severity ${ }^{[30-32,29,33,9,34]}$ or a brief approach consisting of three to four items (i.e., ${ }^{[35-38]}$ ).

Adolescents and young adults highly value friendships and are reliant on their social networks for support ${ }^{[39,40]}$. This means that any disruptions to these social networks can have significant negative impacts even in later life ${ }^{[41]}$. Young people, specifically those around 20 years old have been found to favour the quantity of relationships over the quality of their social interactions, which is in contrast to their older counterparts at age 30 and $50^{[41]}$. This preference may partially explain why young people are more vulnerable to feeling lonely, especially if loneliness may be a consequence of having less meaningful relationships rather than not having more relationships.

Loneliness and gender. The prevalence of loneliness across gender has produced mixed findings. Some studies have shown females often report experiencing feelings of loneliness more than males $[28,32,9]$, particularly when a direct measure of loneliness is used (i.e., 'do you feel lonely?' ${ }^{[31]}$. Although when a comprehensive measure of loneliness involving multiple items that do not directly state the term 'loneliness', Nicolaisen and Thorsen (2014) found males aged 18-29 years old were significantly 
Ionelier compared to females. It is plausible that males may be reluctant to identify with feeling Ionely relative to females, thus report feeling less lonely when asked directly via a single question $[42,31,32]$. However, further research has also observed no differences in loneliness scores across genders ${ }^{[37,29]}$. The varying results may, in part, be due to the different measures used to assess loneliness across studies, however investigations utilising both methods is needed to examine the presence of gender differences in adolescents and young adults experiencing loneliness ${ }^{[8]}$.

Loneliness and other sociodemographic factors. Current research in adolescent and young adult populations have also reported differing results on the experience of loneliness in vulnerable population groups. Some studies have reported greater feelings of loneliness were strongly associated with ethnic minority status and immigrants, receiving financial disability support, unemployment, living alone and ongoing mental illness ${ }^{[43,44]}$. In particular, an Australian study exploring ethnicity in students aged 8 to 17 years old found students from a minority ethnic background (i.e., student and/or parent(s) born in a non-English speaking country) reported feeling lonelier compared with students from majority ethnic groups ${ }^{[45]}$. Additionally, Lasgaard et al. ${ }^{[43]}$ conducted a population-based study on loneliness in Denmark with individuals aged from 16 to 102 years old and reported females aged 16 to 29 years old who resided within a village or in the country were at a greater risk of feeling lonely. However, recent research in the UK has observed no difference in loneliness scores across different socioeconomic groups at the age of 18 years ${ }^{[37]}$. Within an Australian context, it appears differences in loneliness across ethnic groups may be observed, but it remains undetermined as to whether loneliness varies across socioeconomic status.

\section{Factors contributing to loneliness}

There are multiple reasons why adolescents and young adults may be vulnerable to problematic levels of loneliness. Adolescence and young adulthood is characterised by crucial developmental transitions such as identity formation, coping with life transitions (e.g., new school or commencing employment), developing intimate relationships, and growing autonomy from parents ${ }^{[46,26,47]}$. These transitions are likely to trigger the experience of loneliness and may be further exacerbated if the young person does not develop the appropriate skills fundamental to building strong relationships ${ }^{[26]}$. According to the current theories in loneliness, one's capacity to experience positive affect as a way to signal openness to connect with others ${ }^{[48]}$, as well as one's ability to regulate emotions (often referred to as emotion regulation ${ }^{[49]}$ ) may influence a person's capacity to have meaningful and positive social interactions and relationships. 
Affect. Affect is a continuous experience that varies in intensity over time (e.g., energy and lethargy) as opposed to emotions which are the response to an external stimulus (e.g., feeling proud of our child's first words) ${ }^{[50]}$. Positive affect (PA) is defined as the degree to which an individual feels alert, enthusiastic, and active, and is characterised as a high energy state. A person who reports low positive affect is characterised by a low energy state and may feel sadness and lethargy. Similarly, negative affect (NA) is defined as the degree in which an individual feels mood states such as anger, contempt, and disgust and is characterised by a general distress state. PA and NA are not opposites states and can occur independently and simultaneously ${ }^{[51]}$. Individuals who are lonelier compared with less lonely counterparts have been shown to report lower positive affect (e.g., interested) and more negative affect (e.g., upset; ${ }^{[52-54]}$ ). Indeed, within the current cognition model of loneliness ${ }^{[48]}$, those who are lonelier compared with those who are less lonely, also report less positive affect ${ }^{[55]}$ and fewer prosocial behaviours. This is important because positive affect and prosocial behaviours signal a willingness to connect with others. Proponents of the broaden and build theory have stated that positive emotions such as gratitude can help trigger an upward spiral of positive emotions and improve one's mood and wellbeing ${ }^{[56]}$. Additionally, experiencing and expressing positive emotions also buffers the psychological effects of negative emotions ${ }^{[56]}$.

Emotion Regulation. Advocates of the social-functional approach of emotions have maintained that a person's emotions help coordinate social interactions with others. Specifically, positive internal states and grateful behaviour (e.g., smiling and saying 'thank you') encourages reciprocal prosocial behaviour and openness for social interaction ${ }^{[57,58]}$. College students who are able to share positive experiences engage in hedonic emotion regulation processes, that is they upregulate positive emotions and simultaneously downregulate negative emotions such as loneliness ${ }^{[59]}$. Indeed, those who are lonelier also report more difficulties with regulating their emotions compared with less lonely peers ${ }^{[60,61]}$. Lonelier adults are known to rely on maladaptive regulation strategies, for example suppressing their emotional states, or underutilise adaptive regulation strategies, for example they stop reappraising situations ${ }^{[62]}$. Vanhalst, Luyckx, Van Petegem, and Soenens (2018) found that lonelier adolescents when compared to less lonely peers, were more likely to engage in more maladaptive emotion regulation behaviours, such as catastrophizing about a situation ${ }^{[49]}$.

Taken together, the findings of these studies indicate that those who are lonelier (compared with those who are less lonely) are more likely to report lower positive affect, more negative affect, and more maladaptive emotion regulation strategies, all of which may be detrimental for the development and maintenance of relationships. 


\section{Aims and hypotheses}

The first aim was to examine the prevalence rates of loneliness and social isolation risk in adolescents and young people aged between 12 to 25 living in Victoria, Australia. A psychometrically validated measure of loneliness (i.e., UCLA-LS ${ }^{[10]}$ ) and risk of social isolation (i.e., LSNS-6 ${ }^{[63]}$ ) was used to provide a more accurate representation of loneliness and social isolation in adolescents and young adults. We also examined differences in the prevalence of loneliness and social isolation risk across age, gender, socioeconomic status, and country of birth.

The second aim was to examine the relationship between loneliness and social isolation risk to mental health outcomes including social anxiety and depression in adolescents and young people. In an exploratory analysis, we also examine how problematic levels of loneliness may increase one's likelihood of experiencing anxiety and depression in adolescents and young adults. Consistent with previous evidence, we also expect that loneliness and social isolation will be related even when taking into consideration mental health and demographic variables that are known to influence this relationship.

The third aim was to examine other factors that may contribute to loneliness severity. Two factors were considered based on current research; the influence of positive and negative affect and the ability to regulate emotion. Specifically, it was anticipated that higher loneliness would be associated with lower positive affect and higher negative affect scores. It was also anticipated that higher loneliness would be associated with increased emotion suppression and reduced cognitive reappraisal ability in both adolescents and young adults.

Within exploratory analyses, we also examined age and gender differences across the variables of interest and conducted correlational tests on these variables, in order to determine to clarify any differences across adolescents (under 18) and young people (over 18), or between girls and boys, and young men and women.

\section{Method}

\section{Participants}

Young Victorians aged 12 to 25 years participated in this online study. Table 1 provides an overview of the participant demographics including their country of birth, primary language spoken at their residence, current education and employment status, and their living arrangements. 
Table 1: Demographics of Victorian adolescents and young people

\begin{tabular}{|c|c|c|c|}
\hline & $\begin{array}{l}\text { Under } 18 \\
(N=650)\end{array}$ & $\begin{array}{l}\text { Over } 18 \\
(N=870)\end{array}$ & $\begin{array}{c}\text { Overall } \\
(N=1,520)\end{array}$ \\
\hline Variable & $\mathrm{M}(\mathrm{SD})$ or $\%$ & $\mathrm{M}(\mathrm{SD})$ or $\%$ & $\mathrm{M}(\mathrm{SD})$ or \% \\
\hline Age (years) & $13.75(1.63)$ & $21.38(2.32)$ & $18.12(4.30)$ \\
\hline $12-13$ & $52.62 \%$ & - & $22.50 \%$ \\
\hline $14-17$ & $47.39 \%$ & - & $20.26 \%$ \\
\hline $18-20$ & - & $40.00 \%$ & $22.90 \%$ \\
\hline $21-23$ & - & $36.55 \%$ & $20.92 \%$ \\
\hline $24-25$ & - & $23.45 \%$ & $13.42 \%$ \\
\hline Female & $47.23 \%$ & $75.06 \%$ & $63.16 \%$ \\
\hline \multicolumn{4}{|l|}{ Country of birth } \\
\hline Australia & $90.12 \%$ & $73.45 \%$ & $80.57 \%$ \\
\hline \multicolumn{4}{|l|}{ Language } \\
\hline English & $78.27 \%$ & $67.89 \%$ & $72.33 \%$ \\
\hline \multicolumn{4}{|l|}{ Education status } \\
\hline Primary school & $15.08 \%$ & - & - \\
\hline High school & $79.08 \%$ & $4.60 \%$ & - \\
\hline TAFE & $2.31 \%$ & $10.34 \%$ & - \\
\hline Apprenticeship or traineeship & $0.77 \%$ & $2.30 \%$ & - \\
\hline University (undergraduate) & - & $42.53 \%$ & - \\
\hline University (postgraduate) & - & $12.30 \%$ & - \\
\hline Not studying & $2.62 \%$ & $27.93 \%$ & - \\
\hline \multicolumn{4}{|l|}{ Employment status } \\
\hline Casual & $12.62 \%$ & $31.03 \%$ & $23.14 \%$ \\
\hline Part-time & $6.00 \%$ & $25.37 \%$ & $17.07 \%$ \\
\hline Full-time & $2.46 \%$ & $16.03 \%$ & $10.22 \%$ \\
\hline Volunteer & $3.23 \%$ & $3.92 \%$ & $3.63 \%$ \\
\hline Not working & $75.69 \%$ & $23.64 \%$ & $45.95 \%$ \\
\hline \multicolumn{4}{|l|}{ Housing status } \\
\hline With parents, caregivers, or siblings & $96.46 \%$ & $52.24 \%$ & $71.17 \%$ \\
\hline With friends & $0.62 \%$ & $10.82 \%$ & $6.45 \%$ \\
\hline With romantic partner & $1.69 \%$ & $15.88 \%$ & $9.81 \%$ \\
\hline In shared accommodation & $0.46 \%$ & $14.84 \%$ & $8.69 \%$ \\
\hline Alone & $0.62 \%$ & $4.95 \%$ & $3.09 \%$ \\
\hline Other & $0.15 \%$ & $1.27 \%$ & $0.79 \%$ \\
\hline \multicolumn{4}{|l|}{ Socioeconomic index for areas } \\
\hline Most disadvantaged & $48.10 \%$ & $51.90 \%$ & - \\
\hline Somewhat disadvantaged & $44.59 \%$ & $55.41 \%$ & - \\
\hline Neither advantaged or disadvantaged & $44.49 \%$ & $55.51 \%$ & - \\
\hline Somewhat advantaged & $42.78 \%$ & $57.22 \%$ & - \\
\hline Most advantaged & $44.04 \%$ & $55.96 \%$ & - \\
\hline
\end{tabular}




\section{Measures}

Demography. Data including, age, gender, country of birth, language spoken at home, education status, employment status, and housing status were all measured. We also asked for postcodes and coded these according to the socioeconomic index for areas (SEIFA; ${ }^{[64]}$ ) as a measure of socioeconomic status. Specifically, we used the index of relative socioeconomic advantage and disadvantage (IRSEAD) and divided this into 5 quintiles, from 1 (most disadvantaged) to 5 (most advantaged).

UCLA Loneliness Scale - Version 3 (UCLA-LS; ${ }^{[10]}$ ). The UCLA-LS is a 20-item measure consisting of positively and negatively worded items to assess loneliness (e.g., How often do you feel that you are 'in tune' with the people around you?). The items are rated on a 4-point Likert scale from 1 (never) to 4 (always), with higher scores indicating greater feelings of loneliness. The UCLA-LS was found to demonstrate good to excellent reliability $(\alpha=.89-.94)$ and acceptable test-retest reliability $(\alpha=.73$; ${ }^{[10]}$ ). The overall sample internal consistency scores for the UCLA-LS was excellent $(\alpha=.94)$. Full results for the scale responses are presented in Appendix A.

Lubben Social Network Scale - 6 (LSNS-6; ${ }^{[63]}$ ). The LSNS-6 is a six-item short form measure to assess social isolation. There are two subscales relating to: a) people connected by birth, marriage or adoption (e.g., how many relatives do you see or hear from at least once per month?); and b) friends (e.g., how many friends do you see or hear from at least once a month?). Each subscale consists of three-items related to the participant's social network size and perceived physical and emotional supports ${ }^{[63]}$. Items are rated on a 6-point Likert scale from 0 (none) to 5 (nine or more), with scores of 12 or below indicating increased risk for social isolation. The LSNS-6 has demonstrated adequate reliability and good validity ${ }^{[65]}$. The overall sample internal consistency scores for the LSNS-6 was good $(\alpha=.84)$. Full results for the scale responses are presented in Appendix B.

Centre for Epidemiological Studies - Depression (CES-D; ${ }^{[66]}$ ). The CES-D is a 20-item measure that assesses feelings of depression in the past 7 days (e.g., felt sad). Items are rated on a 4-point Likert scale from 0 (less than 1 day) to 3 (5-7 days), with higher scores indicating greater depressive symptomatology. The CES-D is known to have good construct validity and strong reliability ${ }^{[66,67]}$. In adolescent and adult populations, a cut-off score of 20 or more is used to indicate depressive symptom severity which may warrant further investigation ${ }^{[68]}$. The overall sample internal consistency scores for the CES-D was excellent $(\alpha=.94)$. 
Mini-Social Phobia Inventory (Mini-SPIN; ${ }^{[69]}$ ). The Mini-SPIN is a three-item measure of social anxiety derived from the Social Phobia Inventory (SPIN). The three items (e.g., I avoid activities in which I am the centre of attention) are measured on a 5-point Likert scale from 0 (not at all) to 4 (extremely). In adolescent and adult populations, a cut-off score of six or more is recommended to indicate social anxiety symptom severity which may warrant further investigation ${ }^{[69,70]}$. The MiniSPIN has demonstrated good to excellent internal consistency and test-retest reliability over a 4week period ${ }^{[71]}$. The overall sample internal consistency scores for the Mini-SPIN was good $(\alpha=.85)$.

Positive and Negative Affect Schedule - Short Form (PANAS-SF; ${ }^{\left[{ }^{11}\right]}$ ). The PANAS short form measures positive (e.g., enthusiastic) and negative (e.g., upset) affect during the past 7 days. The items are rated on a 5-point Likert type scale from 1 (very slightly or not at all) to 5 (extremely). The PANAS has demonstrated adequate reliability and good convergent validity ${ }^{[51]}$. The overall sample internal consistency scores for the PANAS-SF positive $(\alpha=.82)$ and negative affect $(\alpha=.88)$ subscales was good.

Emotion Regulation Quotient (ERQ; ${ }^{[72]}$ ). The ERQ is a 10 -item measure assessing an individual's tendency to regulate their emotions via two emotion regulation strategies: i) cognitive reappraisal (CR; e.g., When I want to feel less negative emotion, I change the way I'm thinking about the situation), and ii) expressive suppression (ES; e.g., I keep my emotions to myself). Items are scored on a 7-point Likert scale from 1 (strongly disagree) to 7 (strongly agree), with higher scores indicative of greater use of the CR or ES emotion regulation strategies. The ERQ demonstrates acceptable internal consistency ${ }^{[72]}$. The young adult sample internal consistency scores ranged from acceptable to good for the ES $(\alpha=.73)$ and CR $(\alpha=.85)$ subscales, respectively.

Emotion Regulation Quotient for Children and Adolescents - Revised Version (ERQ-CA; ${ }^{[73]}$ ). The ERQ-CA is tailored to individuals aged 12 to 17 years old to ensure age-appropriate language was used (e.g., I keep my feelings to myself). Similar to the ERQ, it is used to measure emotion regulation and is made up of CR and ES emotion regulation strategy subscales. Items are rated on a 5-point Likert scale from 1 (strongly disagree) to 5 (strongly agree). The ERQ-CA demonstrates acceptable and good internal consistency for the ES and CR subscales respectively ${ }^{[72,73]}$. The adolescent sample internal consistency scores ranged from acceptable to good for the ES $(\alpha=.77)$ and CR $(\alpha=.82)$ subscales, respectively. For the purposes of analyses, we have standardized the emotion regulation scores across two samples using the $Z$ score. 


\section{Procedure}

Human research ethics approval was obtained from the university ethics board. External cross-panel market research providers were used to recruit participants from existing databases. Participants under 18 years were invited into the online study via targeted emails sent to parents or legal guardians of adolescents aged 12 to 17 years old who resided in Victoria, Australia. The email described the content and estimated duration of survey and reimbursement provided. Parent or legal guardian consent was obtained to allow for the participant under 18 to complete the online study via the external cross-panel market research providers. If the adolescent was with the parent or legal guardian when consent was provided for their participation, the adolescent could commence the survey. If the adolescent was available at a later time, a follow up email was sent to the parent or legal guardian inviting the adolescent to complete the survey. A similar procedure was employed for participants over 18 years who received an email inviting them to participate in the online study if they were aged 18 to 25 years and resided in Victoria, Australia. Participants over 18 were consented into the online study by the external cross-panel market research providers. Human research ethics approval was obtained for the study.

\section{Data analytic procedure}

Data screening was conducted to ensure the usability of the data. A data integrity script was run to determine whether participants had variation across their scores for all measures. This script involved running a frequency analysis for each participant's responses to ensure that they did not select one response type across multiple scales. In addition, participants' completion time was used to determine whether accurate responses were provided. Participants who completed the study in a rapid time (i.e., 4 minutes or less) were removed from the dataset. This threshold was used because it reflected the completion time from the survey testing phase, and due to the nature of the recruitment, participants are familiar with the platform. In total, 261 participants were removed from the dataset before analysis. For the remaining participants, total scores were created for each variable of interest. Descriptive statistics were calculated for all variables. Overall analyses were conducted in the first instance and were then followed up with separate analyses for young adults and adolescents.

Consistent with previous research, we identified problematic levels of loneliness via scores that fall more than 52 or higher on the UCLA-LS scale. This cut off score was based on the previous Australian Psychological Society Report ${ }^{[7]}$, and consistent with the published mean scores of loneliness severity in young people who have been diagnosed with a serious mental illness ${ }^{[74,75]}$. This method was 
selected given there were no published population norms for the UCLA-LS loneliness measure within the Australian context to compare against.

Mean scores were used for all variables with the exception of the ERQ, this is because two different forms were used and could not be compared between groups without data transformations. Raw scores on these scales were converted to z-scores for analysis. Furthermore, age was further categorised into groups to ensure adequate statistical power to conduct group comparisons.

To determine the strength of the relationship between loneliness and social isolation, Pearson correlation coefficients were calculated. Additionally, hierarchical regressions were conducted to examine whether social isolation predicted loneliness, above and beyond known loneliness correlates such as social anxiety and depression symptom severity. To determine whether adolescents and young adults responded differently to each of the measures, group comparisons were made using standard and Welch's $t$-tests and analyses of variance (ANOVAs). Welch's $t$-tests were conducted for comparisons in instances where Levene's test of equal variances was violated. Bonferroni corrections were made to control for Type 1 error.

\section{Results}

\section{Prevalence of loneliness and social isolation risk}

Prevalence of loneliness was measured using the UCLA-LS total score, and we also included two single item indicators to supplement our understanding of prevalence. First, the UCLA-LS single item, 'How often do you feel alone?' and the CES-D single item, 'How often do you feel lonely?'

UCLA-LS Total: Overall, young Victorians reported a mean loneliness score of 45.27 out of 80 , with a standard deviation of 10.53 . Those that scored above 52 on the UCLA total score were classified as having problematic levels of loneliness and more than one in four (28.12\%) young people scored 52 or above. Specifically, one in six adolescents $(15.80 \%)$ and one in three $(37.10 \%)$ young adults scored above 52, indicating problematic levels of loneliness. As seen in Figure 1 , adolescents $(M=41.73, S D$ $=10.07)$ reported significantly lower levels of loneliness than young adults $(M=47.86, S D=10.10)$, $t(1360.08)=11.59, p<.001$, Cohen's $d=0.61$. 


\section{Loneliness}

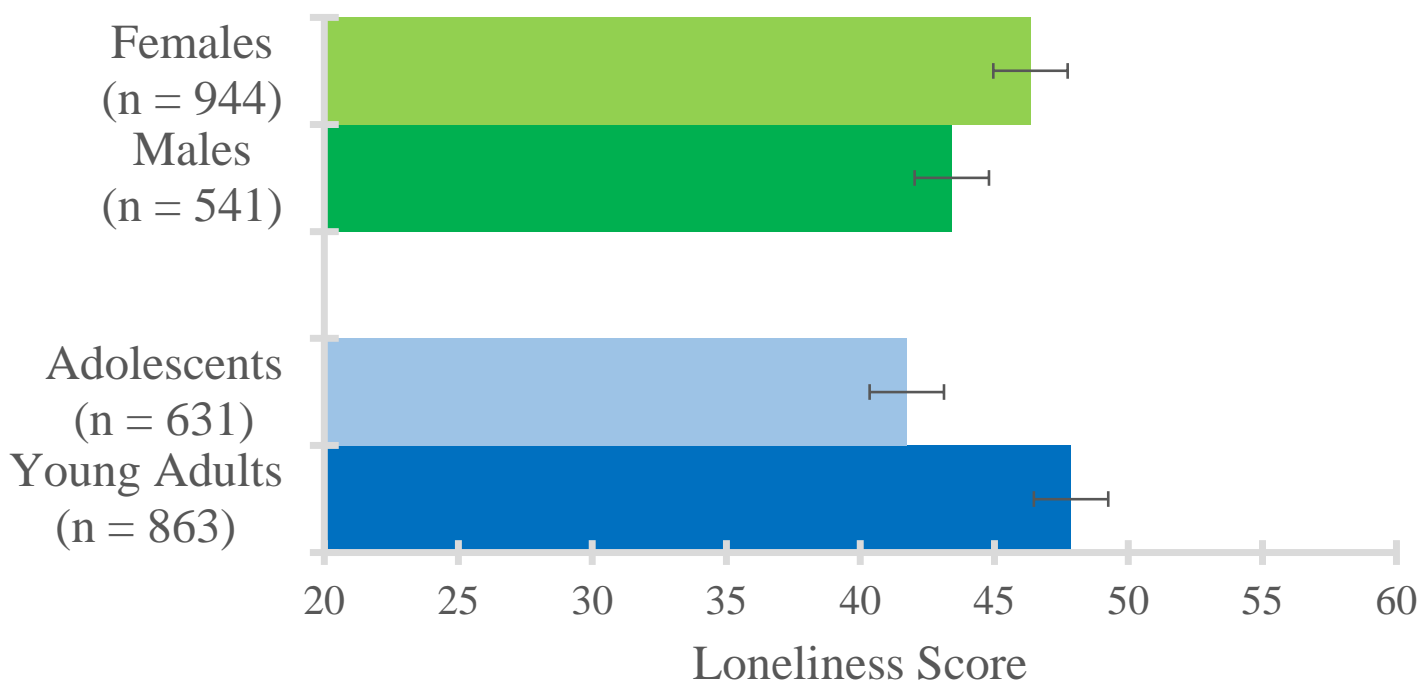

Figure 1. UCLA loneliness scale total scores across gender and age group cohort. Error bars indicate standard error.

UCLA-LS Item 4 How often do you feel alone?: A series of frequency analyses were conducted to determine how often participants felt alone. Overall, approximately one in two young people (55.18\%) from the sample reported they felt alone either sometimes or often. Specifically, two in five adolescents (43.19\%), and two in three young adults (64.09\%) reported they felt alone either sometimes or often.

CES-D Item 14 How often do you feel lonely?: Frequency analysis on the entire dataset revealed that one in four (25.68\%) young people reported feeling lonely for three or more days within the last week. Specifically, one in seven adolescents (12.73\%) and one in three young adults (35.29\%) reported feeling lonely three or more times a week. One in two young people (52.22\%) reported feeling lonely at least once in the last week, specifically, one in three for adolescents (37.89\%), and three in five for young adults (62.86\%) reported feeling lonely at least once per week.

Social isolation risk: Approximately one in three young people (32.14\%), overall, were at risk of social isolation when we used the LSNS-6 total score. Adolescents (27.26\%) reported less socially isolation risk compared with young adults (35.77\%).

Social contact: how many relatives/friends do you see or hear from at least once a month? We also examined the first and fourth items of the LSNS as a measure of social contact. Welch's $t$-tests were used to test whether adolescents and young adults differed between their amount of social contact 
with friends and family. As seen in Table 2 , adolescents $(M=3.14, S D=1.22)$ reported significantly more contact with their family $(M=2.95, S D=1.17), t(1357.94)=3.02, p=.002$, and more contact with friends $(M=3.50, S D=1.25)$ compared with young adults $(M=3.07, S D=1.28), t(1516)=6.51$, $p<.001$.

Table 2: Comparison of loneliness and social isolation risk across adolescents and young adults

\begin{tabular}{|c|c|c|c|c|c|c|}
\hline Variable & $\begin{array}{l}\text { Under } 18 \\
M(S D)\end{array}$ & $\begin{array}{l}\text { Over } 18 \\
M(S D)\end{array}$ & $\begin{array}{l}\text { Overall } \\
M(S D)\end{array}$ & $t$ & $p$ & Cohen's $d$ \\
\hline \multicolumn{7}{|l|}{ UCLA-LS } \\
\hline UCLA-LS Total & $41.73(10.07)$ & $47.86(10.10)$ & $45.27(10.53)$ & -11.59 & $<.001^{* * *}$ & -0.61 \\
\hline \multicolumn{7}{|l|}{ UCLA LS Item 4} \\
\hline $\begin{array}{r}\text { How often do you feel } \\
\text { alone?a }\end{array}$ & $2.26(0.82)$ & $2.68(0.78)$ & $2.50(.83)$ & -9.90 & $<.001^{* * *}$ & -0.52 \\
\hline \multicolumn{7}{|l|}{ CES-D Item 14} \\
\hline $\begin{array}{r}\text { How often do you feel } \\
\text { lonely?b }\end{array}$ & $0.55(0.83)$ & $1.11(1.05)$ & $0.87(1.00)$ & -11.56 & $<.001 * * *$ & -0.59 \\
\hline \multicolumn{7}{|l|}{ LSNS-6 } \\
\hline LSNS-6 Total & $15.95(5.68)$ & $14.56(5.41)$ & $15.15(5.57)$ & 4.84 & $<.001^{* * *}$ & 0.25 \\
\hline Family & $7.73(3.24)$ & $7.03(3.07)$ & $7.33(3.16)$ & 4.29 & $<.001^{* * *}$ & 0.22 \\
\hline Friends & $8.25(3.30)$ & $7.53(3.36)$ & $7.84(3.35)$ & 4.10 & $<.001 * * *$ & 0.22 \\
\hline
\end{tabular}

Note: UCLA-LS = University of California Los Angeles - Loneliness Scale, LSNS-6 = Lubben Social Network Scale 6 items with higher scores indicating reduced risk of social isolation, a UCLA-LS Item 4, range 1 (Never) - 4 (Always). b CES-D Item 14, range 0 (Less than a day) -3 (5 to 7 days). ${ }^{*}=p<.05,{ }^{* *}=p<.01,{ }^{* * *}=p<.001$.

Loneliness, social isolation risk, and demographic factors: A series of analyses were conducted to determine the role of different demography on loneliness and social isolation. Age and gender were investigated in detail, while country of birth, employment, study status, language other than English, and SEIFA scores were presented descriptively (see Appendices A-I).

Loneliness and age: To ensure that there were comparable numbers and homogeneity of variance within each age group, we categorised participants into five age groups; group 1 (12-13 years), group 2 (14-17 years), group 3 (18-20 years), group 4 (21-23 years), and group 5 (24-25 years). A one-way ANOVA with loneliness as the dependent measure was conducted which revealed a significant main effect of age, $F(4,1485)=35.28, p<.001$. This was followed up with Bonferroni corrected mean comparisons to determine any differences between the age groups $(p<.01)$. Twelve to thirteen-year-old participants reported equivalent levels of loneliness with the 14-17-year-old participants. Similarly, 18-20-year-old participants, 21-23 and 24-25-year-old participants, were all equally lonely. 
However, there was a significant difference between adolescents (those under 18 years of age) and young adults (those above 18 years of age). See Figure 5 below. Significant differences were observed between the 12-13-year-old participants and the 18-20, 21-23, and 24-25-year-old age groups (all $p s<.001$ ), such that all the 12-13-year-old participants reported significantly lower levels of loneliness compared with the young adult age groups. Similarly, the 14-17-year-old participants were also significantly less lonely than the young adult age groups.

Social isolation risk and age: An effect of LSNS was also found, $F(4,1498)=5.983, p<.001$, and two adolescent age groups (12-13-year-olds and 14-17-year-olds) reported lower social isolation risk compared with the young adults age groups. In this case, there was a decline in social network scores, indicating an increasing risk for social isolation after the age of 18. See Figure 6 below.

\section{Loneliness}

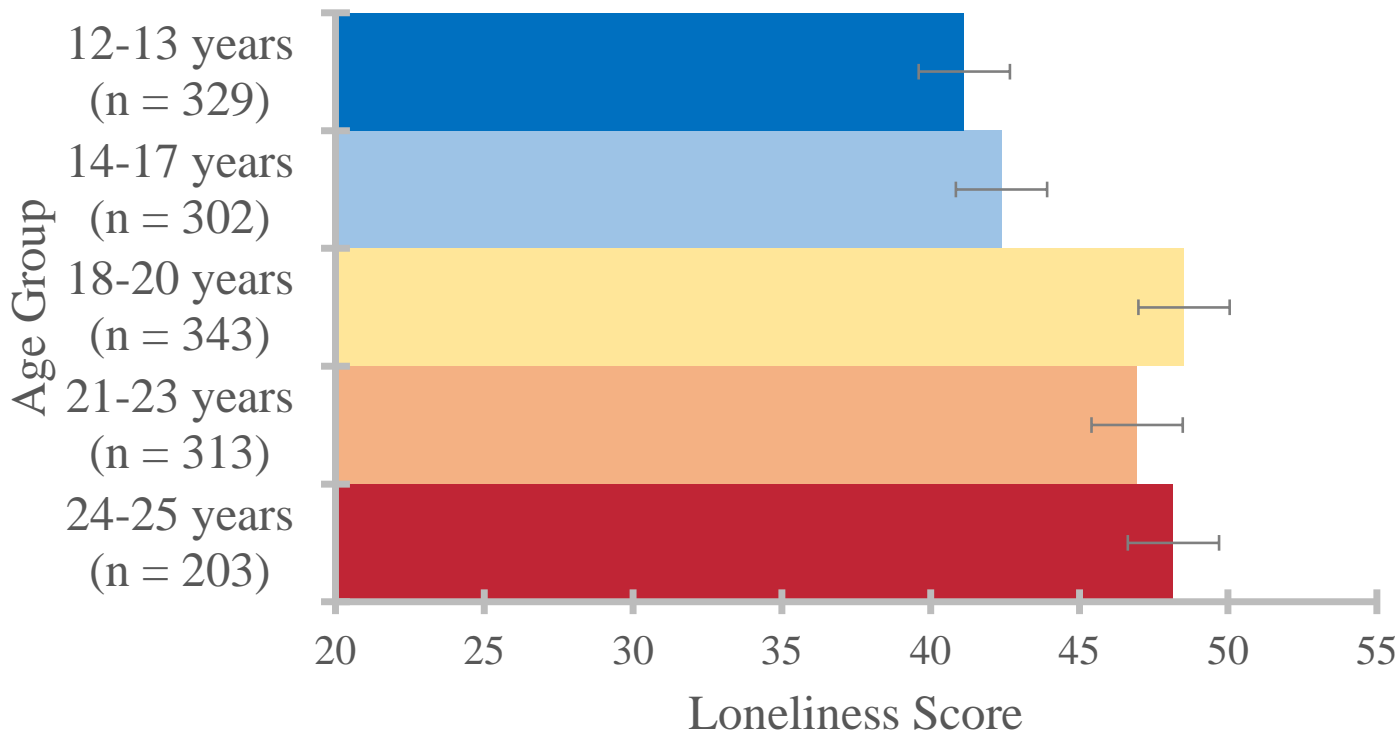

Figure 5. Mean loneliness scores across the five age groups. Higher scores indicate more severe loneliness. Error bars represent standard error. 


\section{Risk for Social Isolation}

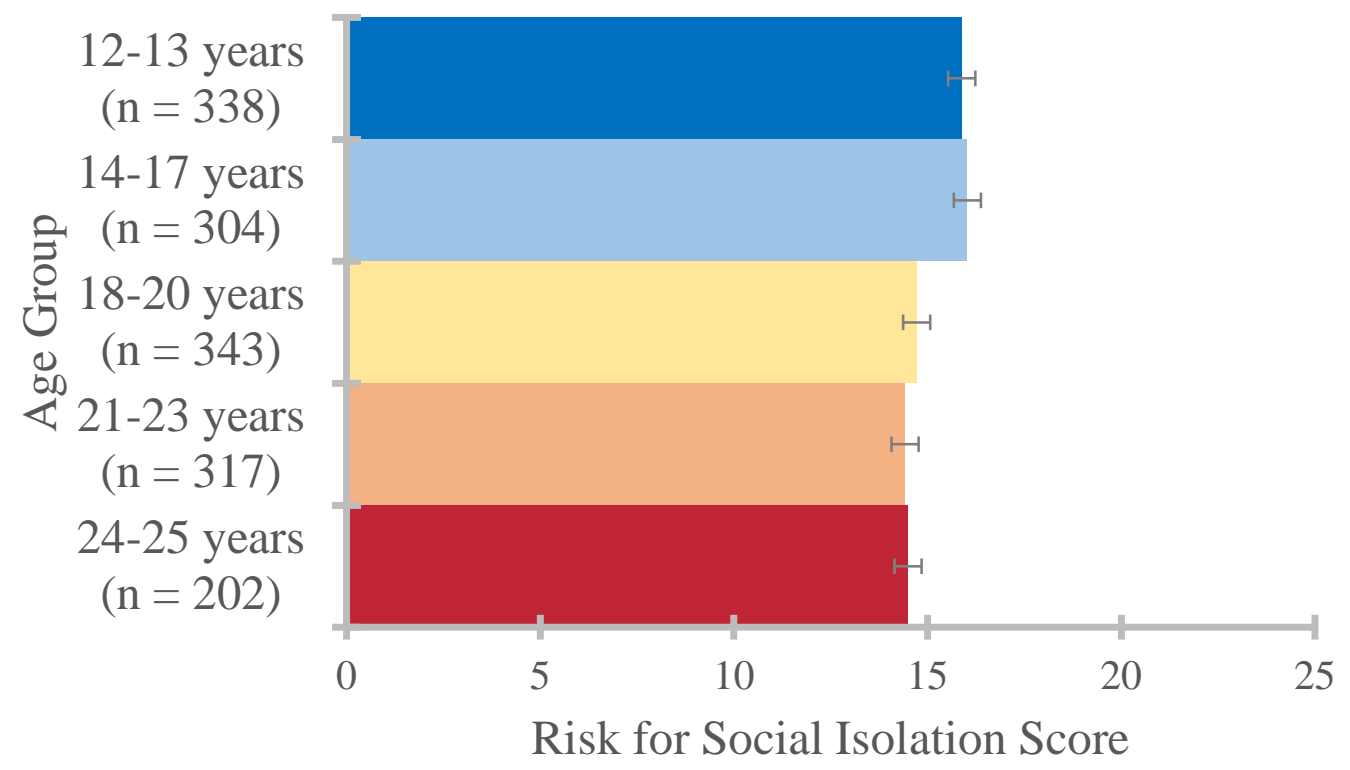

Figure 6. Mean social isolation risk scores across the five age groups. Lower scores indicate a greater risk for social isolation. Error bars represent standard error.

Gender: Overall, a Welch's $t$-test, revealed that males $(M=43.41, S D=10.12)$ reported significantly less loneliness than females $(M=46.35, S D=10.62), t(1169.57)=-5.28, p<.001$. To determine whether this gender effect was similar across adolescents and young adults, we conducted the same analysis separately and results indicated that this effect was driven by young adult males, who reported significantly less loneliness than young adult females. There were no reported differences between males and females on risk for social isolation.

Table 3: Gender differences across loneliness and social isolation risk

\begin{tabular}{lllllll}
\hline & Measure & Males & Females & \multirow{2}{*}{ Cohen's d } \\
& & M(SD) & M(SD) & & \multicolumn{2}{c}{. } \\
\hline Adolescents & UCLA-LS & $41.57(9.92)$ & $41.95(10.24)$ & -0.47 & .639 & -0.04 \\
& LSNS-6 & $15.80(5.40)$ & $16.13(5.98)$ & -0.74 & .459 & -0.06 \\
Young adults & UCLA-LS & $46.33(9.76)$ & $48.37(10.17)$ & -2.55 & $.011^{*}$ & -0.20 \\
& LSNS-6 & $14.91(5.91)$ & $14.43(5.24)$ & 1.03 & .302 & -0.09 \\
\hline
\end{tabular}

Note: UCLA-LS = University of California Los Angeles - Loneliness Scale, LSNS-6 = Lubben Social Network Scale 6 items with higher scores indicating reduced risk of social isolation, ${ }^{*}=p<.05, * *=p<.01, * * *=p<.001$.

\section{Mental health outcomes}

We examined the severity of mental health symptom based on published cut off scores for social anxiety (MINI-SPIN cut off score of 6 or more ${ }^{[69,70]}$ ) and depression (CES-D cut off score of 20 or 
more $\left.{ }^{[68]}\right)$. These scores are indicative of possible problems but are not used to indicate clinical diagnosis (which can only be determined by structured diagnostic tools and interviews).

Social anxiety. Almost one in two young people (47.42\%) met criteria for possible problematic social anxiety. Specifically, one in two young adults $(58.03 \%)$ and one in three adolescents $(31.68 \%)$ met criteria for possible problematic social anxiety.

Depression. Approximately one in two young people (50.10\%) met criteria for possible problematic depressive symptoms. Approximately, two in three young adults (64.2\%) and one in three adolescents (30.80\%) met threshold for possibly having depression.

There was a significant difference between adolescents (those under 18 years of age) and young adults (those above 18 years of age) for social anxiety, $F(4,1507)=37.46, p<.001$ and depression scores, $F(4,1481)=52.55, p<.001$. See Table 4, and Figures 7 and 8 .

Table 4: Comparisons of depression and social anxiety across adolescents and young adults

\begin{tabular}{lcccccc}
\hline & Under 18 & Over 18 & Overall & & & \multirow{2}{*}{ Cohen's $d$} \\
\hline Variable & $\mathrm{M}(\mathrm{SD})$ & $\mathrm{M}(\mathrm{SD})$ & $\mathrm{M}(\mathrm{SD})$ & $t$ & $p$ & -0.74 \\
Mini-SPIN & $13.24(11.53)$ & $22.26(12.72)$ & $18.48(13.11)$ & -14.26 & $<.001^{* * *}$ & -0.62 \\
\hline
\end{tabular}

Note: CES-D = Centre for Epidemiological Studies-Depression, Mini-SPIN = Mini-Social Phobia Inventory.

\section{Depression}

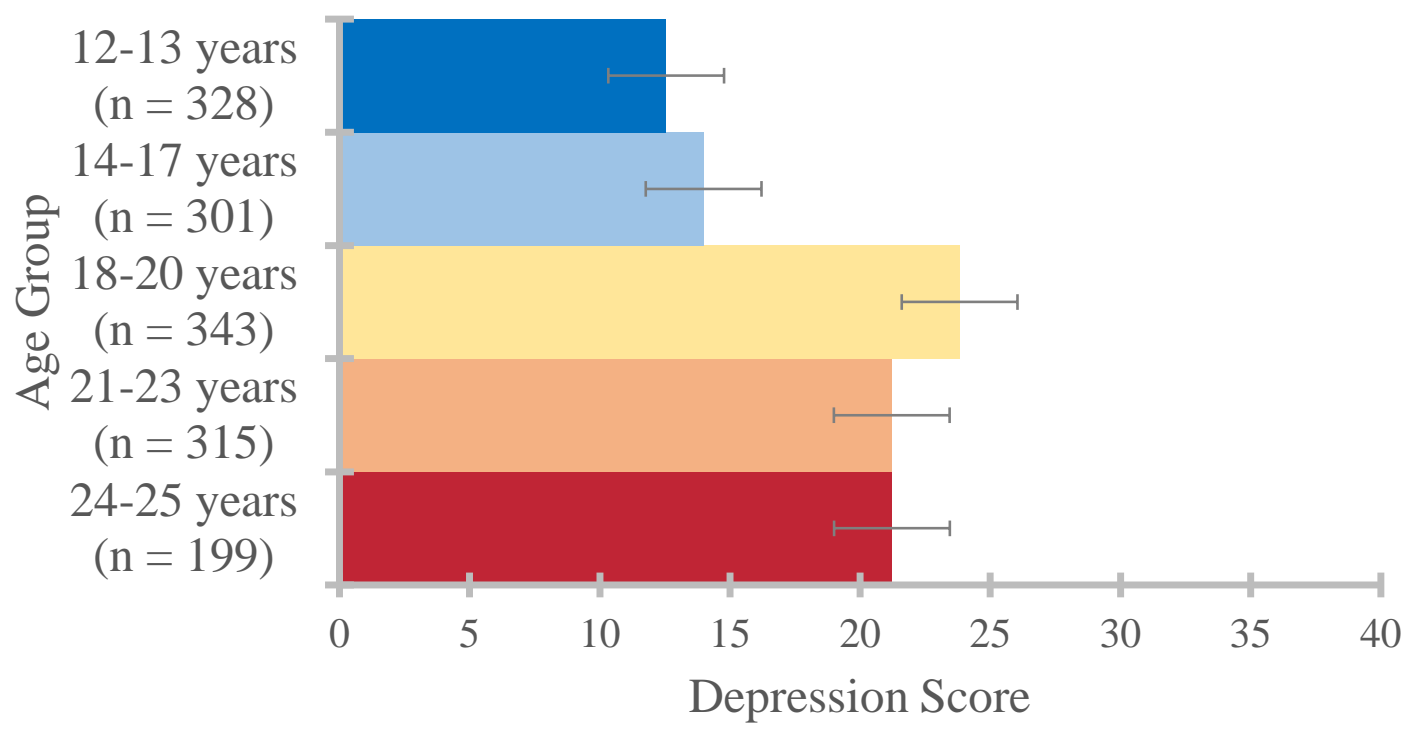

Figure 7. Mean depression scores across the five age groups. Higher scores indicate more severe depressive symptoms. Error bars represent standard error. 


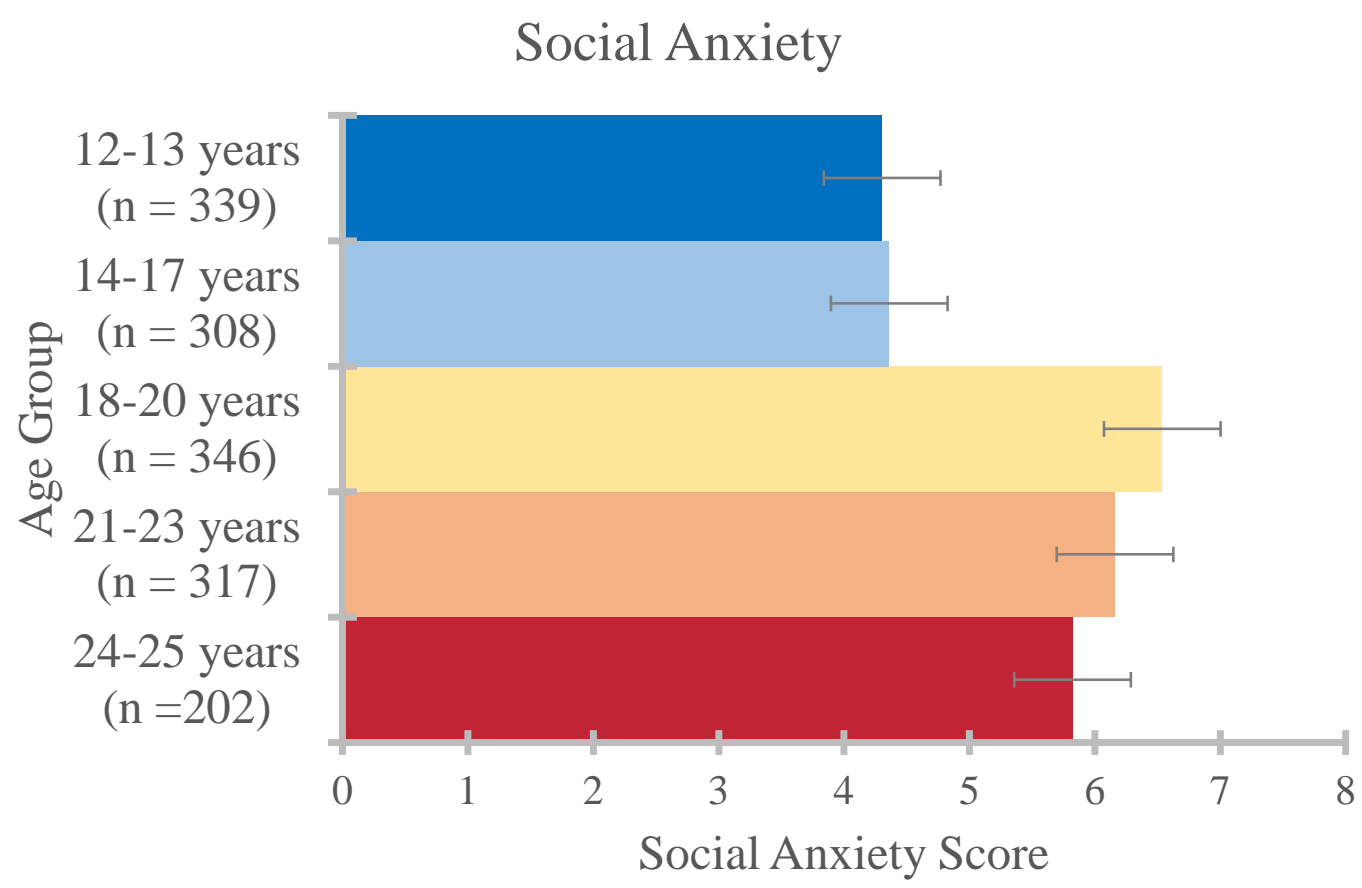

Figure 8. Mean social anxiety scores across the five age groups. Higher scores indicate more severe social anxiety. Error bars represent standard error.

Odds Ratio Analyses: Overall, higher loneliness scores were associated with an increased likelihood of experiencing problematic levels of social anxiety (OR $=1.115,95 \% \mathrm{Cl} 1.100-1.130, p<.001)$ and problematic depression (OR $=1.179,95 \% \mathrm{Cl} 1.158-1.200, p<.001)$. In adolescents, higher loneliness was associated with an increased likelihood of experiencing problematic levels of social anxiety (OR $=1.116,95 \% \mathrm{Cl} 1.09-1.14, p<.001)$ and an increased likelihood of experiencing problematic levels of depression $(\mathrm{OR}=1.195,95 \% \mathrm{Cl} 1.16-1.24, p<.001)$. The same pattern was observed in young adults, whereby higher loneliness was associated with an increased likelihood of experiencing problematic levels of social anxiety $(\mathrm{OR}=1.102,95 \% \mathrm{Cl} 1.08-1.12, p<.001)$ and an increased likelihood of experiencing problematic levels of depression $(\mathrm{OR}=1.157,95 \% \mathrm{Cl} 1.13-1.18, p<.001)$.

Gender differences mental health outcomes. Young adolescent males reported significantly less social anxiety than young adolescent females but this effect size was small. For young adults, males reported significantly less, depression symptoms, social anxiety symptoms, than young adult females (see Table 5). 
Table 5: Gender differences across depression and social anxiety

\begin{tabular}{ccccccc}
\hline & Measure & Males & Females & \multirow{2}{*}{ Cohen's $d$} \\
& & $M(S D)$ & $M(S D)$ & & $p$ & -0.02 \\
\hline Adolescents & CES-D & $13.15(11.48)$ & $13.36(11.62)$ & -0.24 & .813 & -0.16 \\
& Mini-SPIN & $4.12(2.82)$ & $4.58(2.94)$ & -2.04 & $.042^{*}$ & -0.27 \\
Young adults & CES-D & $19.72(11.73)$ & $23.09(12.95)$ & -3.51 & $.001^{* *}$ & -0.27 \\
& Mini-SPIN & $5.33(3.04)$ & $6.54(3.20)$ & -4.83 & $<.001^{* * *}$ & -0.39 \\
\hline
\end{tabular}

Note: $C E S-D=$ Centre for Epidemiological Studies-Depression, Mini-SPIN $=$ Mini-Social Phobia Inventory $*=p<.05, * *=p<$ $.01, * * *=p<.001$.

\section{Affect and emotion regulation}

Affect: Positive and negative affect were measured across age groups. As seen in Table 6, adolescents reported significantly higher positive affect compared with young adults. Adolescents also reported lower negative affect compared with young adults.

Emotion regulation: Both adolescents and young people reported the use of more adaptive emotion regulation strategies such as cognitive reappraisal as opposed to more maladaptive coping strategies such as expressive suppression. Inferential analyses cannot be conducted between the two age groups because using z-transformations standardises the different groups to a normal distribution with each corresponding mean set as 0.00 and standard deviation as 1.00 .

Table 6: Comparisons of affect and emotion regulation across adolescents and young adults

\begin{tabular}{|c|c|c|c|c|c|c|}
\hline & Under 18 & Over 18 & Overall & & & \\
\hline Variable & $\mathrm{M}(\mathrm{SD})$ & $\mathrm{M}(\mathrm{SD})$ & $\mathrm{M}(\mathrm{SD})$ & $t$ & $p$ & Cohen's $d$ \\
\hline \multicolumn{7}{|l|}{ PANAS - SF } \\
\hline Positive Affect & $15.84(4.05)$ & $14.22(4.18)$ & $14.86(4.19)$ & 7.56 & $<.001 * * *$ & 0.39 \\
\hline Negative Affect & $9.35(3.85)$ & $12.73(4.65)$ & $11.28(4.64)$ & -15.43 & $<.001 * * *$ & -0.79 \\
\hline \multicolumn{7}{|l|}{ ERQ } \\
\hline Cognitive Reappraisal & - & $26.75(6.57)$ & - & & & \\
\hline Expressive Suppression & - & $16.60(4.85)$ & - & & & \\
\hline \multicolumn{7}{|l|}{ ERA-CA } \\
\hline Cognitive Reappraisal & $19.83(3.54)$ & - & - & & & \\
\hline Expressive Suppression & $10.94(2.85)$ & - & - & & & \\
\hline
\end{tabular}


Table 7: Adolescent gender differences across affect and emotion regulation factors

\begin{tabular}{ccccccc}
\hline & Measure & Males & Females & & & Cohen's $d$ \\
& & M (SD) & M (SD) & $t$ & $p$ & -0.04 \\
\hline Adolescents & PA & $15.69(4.14)$ & $15.98(3.96)$ & -0.89 & .374 & -0.10 \\
& NA & $9.17(3.75)$ & $9.56(3.92)$ & -1.29 & .197 & -0.03 \\
Young Adults & ERQ - CR & $19.77(3.44)$ & $19.87(2.74)$ & -.33 & .744 & 0.15 \\
& ERQ - ES & $11.13(2.74)$ & $10.71(2.95)$ & 1.84 & .067 & 0.56 \\
& PA & $15.93(4.01)$ & $13.65(4.09)$ & 7.06 & $<.001^{* * *}$ & -0.28 \\
& NA & $11.79(4.26)$ & $13.06(4.75)$ & -3.44 & $<.001^{* * *}$ & 0.25 \\
& ERQ - CR & $27.97(6.35)$ & $26.35(6.61)$ & 3.12 & $.002^{* *}$ & 0.39
\end{tabular}

Note: PA = Positive Affect subscale of the Positive and Negative Affect Schedule, NA = Negative Affect subscale of the Positive and Negative Affect Schedule, ERQ-CR = Emotion Regulation Quotient-Cognitive Reappraisal, ERQ-ES =Emotion Regulation Quotient-Expressive Suppression. ERQ-CR and ERQ-ES have been transformed to z-scores for this analysis because of the different version used for adolescents and young adults. ${ }^{*}=p<.05,{ }^{* *}=p<.01, * * *=p<.001$.

For young adults, males reported significantly less negative affect than females (see Table 7). Conversely, young adult males reported more positive affect than females and employed more cognitive reappraisal and expressive suppression emotion regulation strategies than females.

\section{Relationships between loneliness, social isolation risk, mental health, affect, and emotion regulation in young Victorians}

A series of Pearson correlations tests were conducted to determine the relationships between loneliness, social isolation risk, and mental health variables for both adolescents and young adults separately. In adolescents, higher loneliness was associated with an increased risk of being socially isolated, as well as higher depression, social anxiety, negative affect, and greater use of expressive suppression emotion regulation strategies. Higher loneliness was associated with reduced positive affect and reduced cognitive appraisal strategies. In addition, higher positive affect was significantly associated with fewer expressive suppression strategies, but there was no significant relationship between cognitive reappraisal and expressive suppression. Pearson correlation coefficients for adolescents are in Table 8 below. 
Table 8: Correlations between demographic, loneliness, social isolation, mental health, affect, and emotion regulation variables in adolescents

\begin{tabular}{|c|c|c|c|c|c|c|c|c|c|c|}
\hline & 1 & 2 & 3 & 4 & 5 & 6 & 7 & 8 & 9 & 10 \\
\hline 1 Age & - & & & & & & & & & \\
\hline 2 Gender & .00 & - & & & & & & & & \\
\hline 3 UCLA & .03 & .02 & (.93) & & & & & & & \\
\hline 4 LSNS & .03 & .03 & $-.54 * * *$ & $(.85)$ & & & & & & \\
\hline 5 CES-D & .04 & .01 & $.59 * * *$ & $-.30 * * *$ & (.93) & & & & & \\
\hline 6 SPIN & -.01 & $.08^{*}$ & $.53 * * *$ & $-.29 * * *$ & $.44^{* * *}$ & $(.83)$ & & & & \\
\hline $7 \mathrm{PA}$ & $-.08 *$ & .04 & $-.55 * * *$ & $.40 * * *$ & $-.39 * * *$ & $-.34 * * *$ & $(.84)$ & & & \\
\hline $8 \mathrm{NA}$ & .02 & .05 & $.58 * * *$ & $-.29 * * *$ & $.65 * * *$ & $.50 * * *$ & $-.22 * * *$ & $(.87)$ & & \\
\hline $9 \mathrm{CR}$ & .07 & .01 & $-.23 * * *$ & $.10^{* *}$ & $-.18^{* * *}$ & $-.14^{* * *}$ & $.26 * * *$ & $-.16 * * *$ & $(.82)$ & \\
\hline $10 \mathrm{ES}$ & $.12 * *$ & -.07 & $.48 * * *$ & $-.35 * * *$ & $.38 * * *$ & $.33^{* * *}$ & $-.34 * * *$ & $.41 * * *$ & .06 & (.77) \\
\hline
\end{tabular}

Note. Gender is dummy coded 0 for female, 1 for male. UCLA = University of California Los Angeles - Loneliness Scale, LSNS = Lubben Social Network Scale 6 items with higher scores indicating reduced risk of social isolation, CES-D = Centre for Epidemiological Studies-Depression, SPIN = Mini-Social Phobia Inventory, PA = Positive Affect subscale of the Positive and Negative Affect Schedule, NA = Negative Affect subscale of the Positive and Negative Affect Schedule, CR = Cognitive Reappraisal subscale of the Emotion Regulation Quotient (ERQ-CR), ES = Expressive Suppression subscale of the Emotion Regulation Quotient (ERQ-ES). * = $p<.05,{ }^{* *}=p<.01, * * *=p<.001$. Internal consistencies are displayed in parentheses on the diagonal.

For young adults, the general pattern of findings was similar to adolescents with two major exceptions. First, for young adults, gender covaried with almost all factors except for risk for social isolation. Female participants were more likely to report higher loneliness, depression, and social anxiety compared to male participants. Male participants were also more likely to report higher positive affect, cognitive reappraisal, and expressive suppression than female participants. Second, age negatively covaried with negative affect, depression, and social anxiety symptoms, such that the older participants were, the fewer symptoms or negative affect they reported. Young adults who were older reported more positive affect than those who were younger (but over the age of 18). Pearson correlation coefficients can be found in Table 9 below. 
Table 9: Correlations between demographic, loneliness, social isolation, mental health, affect, and emotion regulation variables in young adults

\begin{tabular}{|c|c|c|c|c|c|c|c|c|c|c|}
\hline & 1 & 2 & 3 & 4 & 5 & 6 & 7 & 8 & 9 & 10 \\
\hline 1 Age & - & & & & & & & & & \\
\hline 2 Gender & -.06 & - & & & & & & & & \\
\hline 3 UCLA & -.06 & $.09 *$ & $(.93)$ & & & & & & & \\
\hline 4 LSNS & -.01 & -.04 & $-.59 * * *$ & $(.82)$ & & & & & & \\
\hline 5 CES-D & $-.12^{* *}$ & $.11^{* *}$ & $.64 * * *$ & $-.36^{* * *}$ & (.92) & & & & & \\
\hline $6 \mathrm{SPIN}$ & $-.09 * *$ & $.16^{* * *}$ & $.52^{* * *}$ & $-.32^{* * *}$ & $.44 * * *$ & $(.84)$ & & & & \\
\hline $7 \mathrm{PA}$ & $.07 *$ & $-.23^{* * *}$ & $-.44^{* * *}$ & $.32^{* * *}$ & $-.44^{* * *}$ & $-.28^{* * *}$ & $(.79)$ & & & \\
\hline $8 \mathrm{NA}$ & $-.11^{* *}$ & $.12^{* *}$ & $.52^{* * *}$ & $-.26^{* * *}$ & $.70 * * *$ & $.45^{* * *}$ & $-.28^{* * *}$ & $(.85)$ & & \\
\hline $9 \mathrm{CR}$ & $.11^{* *}$ & $-.11^{* *}$ & $-.30 * * *$ & $.22^{* * *}$ & $-.33^{* * *}$ & $-.19 * * *$ & $.41^{* * *}$ & $-.24 * * *$ & $(.85)$ & \\
\hline $10 \mathrm{ES}$ & -.05 & $-.16^{* * *}$ & $.41^{* * *}$ & $-.32^{* * *}$ & $.31^{* * *}$ & $.29 * * *$ & $-.08^{*}$ & $.25^{* * *}$ & .03 & (.73) \\
\hline
\end{tabular}

Note. Gender is dummy coded 0 for female, 1 for male. UCLA = University of California Los Angeles - Loneliness Scale, LSNS = Lubben Social Network Scale 6 items with higher scores indicating reduced risk of social isolation, CES-D = Centre for Epidemiological Studies-Depression, SPIN = Mini Social Phobia Inventory, PA = Positive Affect subscale of the Positive and Negative Affect Schedule, NA = Negative Affect subscale of the Positive and Negative Affect Schedule, $C R=$ Cognitive Reappraisal subscale of the Emotion Regulation Quotient (ERQ-CR), ES = Expressive Suppression subscale of the Emotion Regulation Quotient (ERQ-ES). ${ }^{*}=p<.05,{ }^{* *}=p<.01,{ }^{* * *}=p<.001$. Internal consistencies are displayed in parentheses on the diagonal.

\section{Relationship between social isolation risk and loneliness}

In both adolescent and young adults, lower social isolation risk was moderately related to lower levels of loneliness ( $r=-.54, p<.001$ and $r=-.57, p<.001$, respectively). To further explore the contribution of social isolation risk and loneliness, we conducted a hierarchical linear regression to determine whether loneliness would be predicted by a person's risk for social isolation even after controlling for known correlates such as mental health symptom severity. At step 1, age, gender, depression, and social anxiety were entered into the model. Variables entered at step 1 significantly explained $50.40 \%$ of the total variance of loneliness, $F(4,1426)=361.79, p<.001$, however only depression and social anxiety were significant unique predictors of loneliness with depression being the strongest predictor $(\beta=.50, p<.001)$ followed by social anxiety $(\beta=.31, p<.001)$. Total LSNS scores were added into the model at step 2 which significantly predicted loneliness, $F(5,1425)=$ 437.43), $p<.001$. Furthermore, risk for social isolation $(\beta=-.35, p<.001)$ predicted an additional $10.20 \%$ of variance in loneliness over and above that of $\operatorname{step} 1, F c h(1,1425)=367.78, p<.001$. This model explained approximately $61 \%$ of the variance in loneliness. 


\section{The contribution of affect and emotion regulation on loneliness}

To help understand why some individuals experience higher levels of loneliness than others, we extended the previous model to determine the influence of positive and negative affect and emotion regulation strategies, including cognitive reappraisal and expressive suppression. At step 1, age, gender, depression, and social anxiety were entered into the model. Step 1 significantly explained $50.50 \%$ of the variance in loneliness, $F(4,1403)=357.62, p<.001$. Depression and social anxiety were both significant unique predictors of loneliness with depression being the strongest predictor $(\beta=.50, p<.001)$ followed by social anxiety $(\beta=.30, p<.001)$. Age and gender failed to uniquely predict loneliness at step 1 . Social isolation scores were added into the model at step 2 . The inclusion of social isolation scores $(\beta=-.35, p<.001)$ significantly predicted loneliness, $F(5,1402)=$ $428.02, p<.001$. At step 2 , the model explained an additional $9.90 \%$ of the variance in loneliness, $\operatorname{Fch}(1,1402)=351.87, p<.001$.

At step 3, positive and negative affect were added to the model. The inclusion of these variables significantly predicted loneliness, $F(7,1400)=340.54$, with both positive affect $(\beta=-.16, p<.001)$ and negative affect $(\beta=.15, p<.001)$ being equally important at predicting loneliness. The inclusion of positive and negative affect significantly predicted an additional $2.60 \%$ of the variance in loneliness, $F c h(2,1400)=48.83, p<.001$. In the final step, cognitive reappraisal and expressive suppression were added into the model, and was found to significantly predict loneliness, $F(9,1398)$ $=283.77, p<.001$. Both cognitive reappraisal $(\beta=-.06, p<.001)$ and expressive suppression $(\beta=.14$, $p<.001)$ uniquely predicted loneliness. The inclusion of these emotion regulation strategies, predicted an additional $1.60 \%$ of variance over and above the other levels, $F c h(2,1398)=32.11, p$ $<.001$. Overall, this model explained approximately $65 \%$ of the variance in loneliness.

\section{Discussion}

Adolescents and young adults are thought to be vulnerable to experiencing loneliness despite being embedded within social structures such as school and work. The primary study aim was to determine the prevalence rates of loneliness in adolescents aged between 12 and 17 years and young adults aged between 18 to 25 years, living in Victoria, Australia. In order to gain a comprehensive understanding of loneliness, we used a comprehensive measure of loneliness, and examined two single item questions. Our findings indicated that the prevalence rates for loneliness were significantly different between adolescents and young adults. Using the UCLA-LS, at least one in four (28.12\%) of young Victorians reported problematic levels of loneliness. Specifically, an estimated one in six adolescents (15.80\%) and one in three $(37.10 \%)$ young adults reported feeling 
problematic levels of loneliness. Overall, around one in two young people reported they felt sometimes or often felt alone, and one in four reported feeling lonely for at least three more days in the last week.

Overall, adolescents reported significantly less loneliness when compared with young adults. Our findings broadly reflect those by Qualter et al. (2015) who reported adolescents were less loney when compared with young adults ${ }^{[8]}$. To our knowledge, only two studies ${ }^{[76,77]}$ have administered the comprehensive 20-item UCLA-LS to examine loneliness. Bruce et al. ${ }^{[76]}$ conducted a national survey exploring loneliness in 20,096 American adults and found that participants aged 18-25 years old reported comparable mean loneliness scores $(M=47.84, S D=9.02)$ to our sample of young adults $(M=47.86, S D=10.10)$. Additionally, Shevlin and colleagues (2013) examined loneliness in a sample of 1,434 adolescents aged 16 years old in Northern Ireland ${ }^{[77]}$. They quantified high loneliness as one standard deviation above their sample mean of $32.82(S D=9.43)$ and noted that $15.6 \%$ of their sample scored over $42^{[77]}$. This is lower than our results, where we obtained a mean of $42.04(S D=10.07)$ in our adolescent sample.

There are two plausible explanations as to why young adults may be significantly lonelier than adolescents. First, adolescents in this sample were more likely to live with family, siblings, and caregivers (96\%) compared with young adults (52.50\%). While living with family does not mean receiving adequate support, having convenient access to family may assist with loneliness severity. Our study was unable to determine whether living status directly influenced loneliness severity or influenced loneliness severity via access to family. Second, young adulthood is also marked by significant life events such as leaving school or home and young adults may also embark on new social challenges such as commencing higher education or work ${ }^{[78]}$. These life events may increase social isolation and therefore contribute to loneliness. Almost half of the young adults (45.30\%) surveyed were undergraduates at university and a further $11.30 \%$ were postgraduates at university. University students are well-known to be particularly vulnerable to experiencing mental ill health $[79,80,41,81]$.

The second study aim was to determine the relationship between social isolation risk and loneliness. We hypothesised and found support that loneliness and social isolation risk would be moderately related. Hence, those who are lonelier are of increased risk of social isolation, compared with those who are less lonely, across both age groups. The relationship between social isolation risk and loneliness remained even after controlling for mental health symptom severity. The risk for social 
isolation continued to predict loneliness, explaining approximately $10 \%$ of the variance. This is consistent with previous research that contact with others and participation in social activities, is moderately associated with functional social indicators, for example, loneliness ${ }^{[82,37]}$.

Findings from our exploratory analyses also indicated that those who are lonelier are at an increased risk of poor mental health. We found that lonelier adolescents and young adults were more likely to develop problematic levels of social anxiety and depression ( $18 \%$ and $12 \%$ respectively). This is consistent with an Australian general adult population, who are $13 \%$ more likely to report problematic social anxiety symptoms and $15 \%$ more likely to report problematic depressive symptoms ${ }^{[7]}$. Specifically, adolescents reported an elevated risk for mental ill health ( $12 \%$ and $20 \%$ for social anxiety and depression respectively) compared to young adults ( $10 \%$ and $16 \%$ for social anxiety and depression respectively).

The third study aim was to examine factors that may contribute to the loneliness severity. We hypothesised that there would be two main factors that contribute to loneliness, affect (positive and negative) and emotion regulation ability. Those who were lonelier compared with those who were less lonely also reported lower positive affect and more negative affect across both adolescents and young adults. The observed relationships between positive affect and loneliness also support the social functional approach of emotions which suggests that increased positive emotions may buffer the impact of loneliness.

Similarly, those who were lonelier compared with those who were less lonely also reported using more adaptive maladaptive techniques to regulate their emotions. The relationships between adaptive (cognitive reappraisal), maladaptive (expressive suppression) emotion regulation strategies and loneliness, are consistent with Kearns and Creaven (2017) and Marroquin et al. (2016) who have previously shown that those who are lonelier reported more difficulties with regulating their emotions ${ }^{[60,61]}$. Hence, it is likely that increasing positive affect and teaching cognitive reappraisal techniques may help mitigate loneliness severity, whereas, reducing negative affect and expressive suppression techniques contribute to more feelings of loneliness. These results provide preliminary evidence for how loneliness can be addressed in adolescents and young people and have practical implications for the way the development of interventions. 


\section{Study limitations}

There are several study limitations. First, participants under 18 were recruited through their parents. At least one parent was already engaged with the cross-panel provider and nominated their adolescent to participate. It is plausible that recruiting through this avenue may bias results and adolescents recruited for this study may hold have higher parental support compared with their same age peers. While parental support was not measured in this study, there is evidence to support that low parental support has been known to be negatively associated with higher loneliness in adolescents ${ }^{[83]}$. Second, data was collected via online surveys and while this method is able to capture more participants in a cost effective and relatively short time frame, we also had a high rate of non-response and had to screen for non-variable responses ${ }^{[84]}$. Third, in order to mitigate dropout during the survey, we opted to administer brief scales over more comprehensive versions. An example is the use of the Mini-SPIN to measure social anxiety as opposed to the more comprehensive Social Interaction Social Anxiety Scale ${ }^{[85]}$. By doing so, we may not have measured social anxiety symptoms comprehensively and inadvertently under estimated its influence on loneliness severity. This is important especially when there is now evidence to indicate that loneliness and social anxiety is not just reciprocal in adults aged 18 to $87^{[6]}$ but also in children and adolescents ${ }^{[86,87]}$. Last, we only captured the associations between loneliness and its relationships to these factors at one timepoint using a cross-sectional study design and this limits our ability to draw inferences to casual relationships.

\section{Future directions}

These findings provide preliminary evidence on the loneliness severity in Victorian adolescents and young people. Young adults were significantly lonelier than adolescents in this study. Furthermore, social isolation risk may play a role in contributing to loneliness even though this relationship is modest. Loneliness increases the likelihood of experiencing poorer mental health outcomes such as depression and social anxiety. We are limited in determining casual inferences due to the crosssectional study design, it is likely that loneliness may be an antecedent to more problematic mental health such as higher depression and social anxiety, consistent with previous work ${ }^{[19,6]}$. Specifically, loneliness may be a feasible primary target within preventative mental health programs. Other contributing factors included the expression of positive affect and one's ability to demonstrate adaptive emotion regulation strategies such as cognitive reappraisal. These skills may further enhance the ability to develop meaningful relationships with others and inadvertently reduce loneliness. 
There are however remaining questions. For example, in this study, there was no gender difference in loneliness for adolescents but there was a significant but small gender difference for those over 18, (i.e., young men reported significantly lower levels of loneliness than young women). Adopting a longitudinal study design will allow us to map the trajectory of loneliness over this crucial development phase. For example, how do the trajectories of boys' and girls' loneliness differ from adolescence to young adulthood?

While addressing loneliness in adolescents and young people is likely to yield more positive health outcomes, there is a lack of evidence-based programs mitigating loneliness as a primary target. It is likely that early intervention programs that can target loneliness in adolescents may also protect adolescents from developing more problematic levels of loneliness, social anxiety, and depression in young adulthood. Educational programs designed to reduce loneliness however may be more effective if they include teaching cognitive reappraisal of social interactions that may be ambiguous, and how to signal a willingness to connect with others via positive affect. Indeed, solutions that adopt a cognitive-behavioural and positive psychology approach have already shown promise in terms of reducing loneliness in young people and other vulnerable groups ${ }^{[74,75,88,1]}$. Additionally, programs that are further augmented with the provision of safe social opportunities are likely to be more effective than those without.

Development of such programs may also benefit from a co-designed approach with consumers to ensure program uptake and engagement, and to reduce attrition ${ }^{[89,90]}$. Programs that are developed in line with latest evidence-based research together with consumer views, should however be evaluated within rigorous research design such as randomised controlled trials in order to measure the acceptability, feasibility, and effectiveness of the program.

\section{Conclusions}

Young adults aged 18 to 25 years old reported significantly more problematic levels of loneliness when compared with adolescents aged 12 to 17 years. Loneliness and social isolation risk are somewhat related but more importantly, loneliness increases the likelihood of poorer mental health outcomes in adolescents and young adults. Additional factors such as increasing positive affect and the use of adaptive emotion regulation strategies may further reduce loneliness. Programs that can modify the way we think and manage about our social relationships as well as those that provide additional safe social opportunities may yield the positive outcomes for adolescents and young people. 


\section{Appendex A}

\section{Responses for the UCLA Loneliness Scale (Revision 3)}

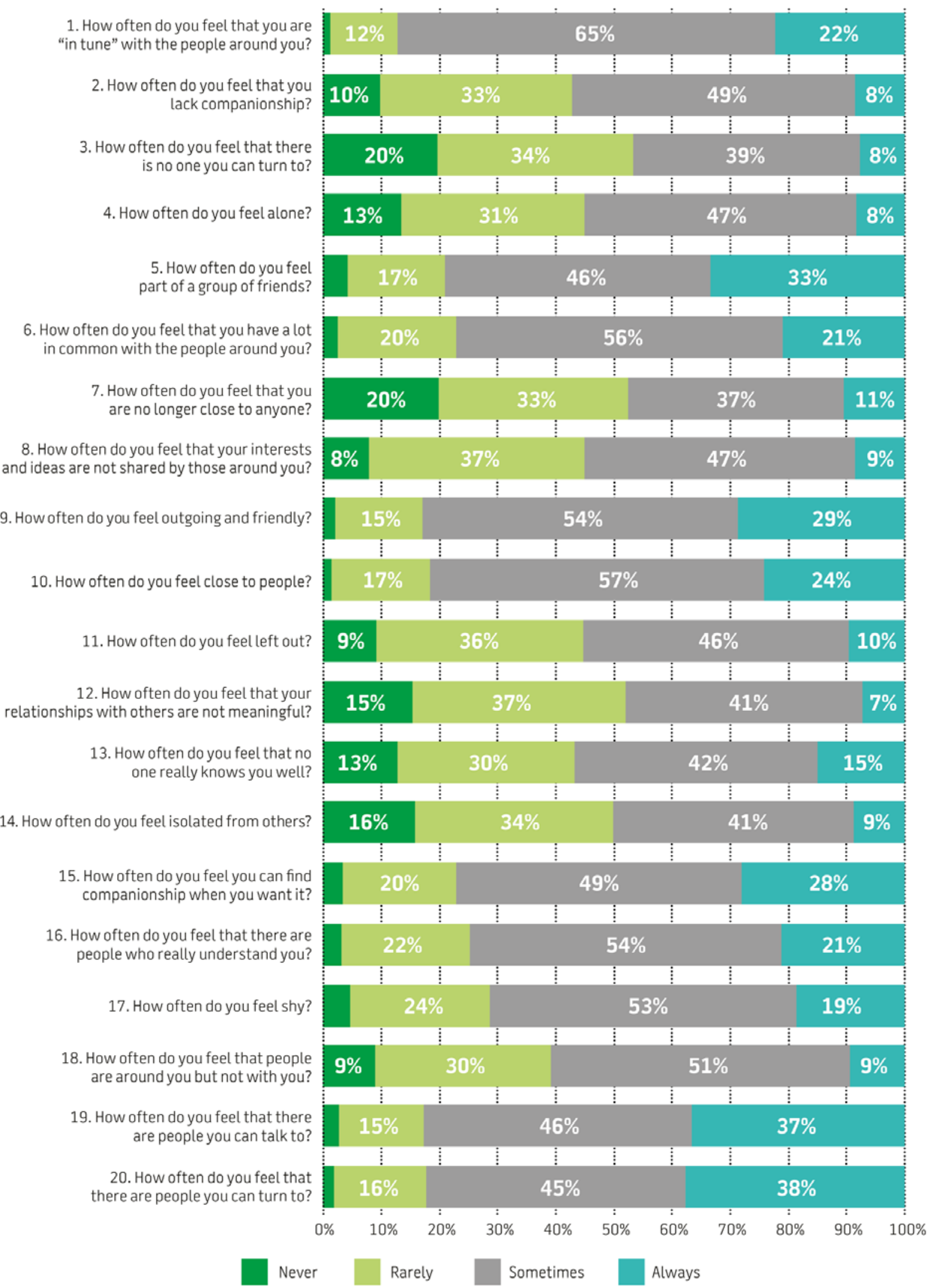

Percentage of respondents $(n=1,490-1,520)$ 


\section{Appendix B}

Responses for the Lubben Social Network Scale (LSNS-6)

\begin{tabular}{|c|c|c|c|c|c|c|c|c|c|}
\hline $\begin{array}{l}\text { 1. How many relatives do you see or } \\
\text { hear from at least once a month? }\end{array}$ & $7 \%$ & \multicolumn{2}{|l|}{$17 \%$} & \multicolumn{3}{|c|}{$38 \%$} & \multicolumn{2}{|c|}{$24 \%$} & $11 \%$ \\
\hline $\begin{array}{l}\text { 2. How many relatives do you feel at ease with } \\
\text { that you can talk about private matters? }\end{array}$ & \multicolumn{2}{|c|}{$16 \%$} & & \multicolumn{3}{|c|}{$30 \%$} & \multicolumn{3}{|c|}{$27 \%$} \\
\hline $\begin{array}{l}\text { 3. How many relatives do you feel close to } \\
\text { such that you could call them for help? }\end{array}$ & $9 \%$ & $16 \%$ & \multicolumn{3}{|c|}{$28 \%$} & \multicolumn{3}{|c|}{$32 \%$} & $10 \%$ \\
\hline $\begin{array}{l}\text { 4. How many friends do you see or } \\
\text { hear from at least once a month? }\end{array}$ & $7 \%$ & $15 \%$ & \multicolumn{3}{|c|}{$31 \%$} & \multicolumn{3}{|c|}{$25 \%$} & $20 \%$ \\
\hline $\begin{array}{l}\text { 5. How many friends do you feel at ease with } \\
\text { that you can talk about private matters? }\end{array}$ & $9 \%$ & $1.7 \%$ & \multicolumn{3}{|c|}{$29 \%$} & \multicolumn{3}{|c|}{$31 \%$} & $9 \%$ \\
\hline \multirow{3}{*}{$\begin{array}{l}\text { 6. How many friends do you feel close to } \\
\text { such that you could call them for help? }\end{array}$} & $10 \%$ & $17 \%$ & \multicolumn{3}{|c|}{$27 \%$} & \multicolumn{3}{|c|}{$30 \%$} & $10 \%$ \\
\hline & & ! & : & : & $\vdots$ & : & $\vdots$ & & $\vdots$ \\
\hline & $10 \%$ & $20 \%$ & $30 \%$ & $40 \%$ & $50 \%$ & $60 \%$ & $70 \%$ & $80 \%$ & $90 \%$ \\
\hline None & & wo & Three & & Five & & or more & & \\
\hline
\end{tabular}

Percentage of respondents $(n=1,503-1,520)$

Each response was rounded up to the nearest whole integer individually, therefore total values may exceed $100 \%$. 


\section{References}

1. Masi, C. M., Chen, H. Y., Hawkley, L. C., \& Cacioppo, J. T. (2011). A meta-analysis of interventions to reduce loneliness. Personality and Social Psychology Review, 15, 219-266. doi:10.1177/1088868310377394

2. Peplau, L., \& Perlman, D. (1982). Perspectives on loneliness. In L. Peplau \& D. Perlman (Eds.), Loneliness: A Sourcebook of Current Theory, Research and Therapy. (pp. 1-20). New York: John Wiley and Sons.

3. Heinrich, L. M., \& Gullone, E. (2006). The clinical significance of loneliness: A literature review. Clinical Psychology Review, 26, 695-718. doi:10.1016/j.cpr.2006.04.002

4. Hawkley, L. C., Burleson, M. H., Berntson, G. G., \& Cacioppo, J. T. (2003). Loneliness in everyday life: Cardiovascular activity, psychosocial context, and health behaviors. Journal of Personality and Social Psychology, 85, 105-120. doi:http://dx.doi.org/10.1037/0022-3514.85.1.105

5. Wilson, R. S., Krueger, K. R., Arnold, S. E., Schneider, J. A., Kelly, J. F., Barnes, L. L., . . Bennett, D. A. (2007). Loneliness and risk of Alzheimer disease. Archives of General Psychiatry, 64, 234-240. doi:10.1001/archpsyc.64.2.234

6. Lim, M. H., Rodebaugh, T. L., Zyphur, M. J., \& Gleeson, J. F. (2016). Loneliness over time: The crucial role of social anxiety. Journal of Abnormal Psychology, 125, 620-630. doi:10.1037/abn0000162

7. Australian Psychological Society \& Swinburne University of Technology (2018). Australian Loneliness Report: a survey exploring the loneliess levels of Australians and the inpact on their health and wellbeing. Retrieved from https://psychweek.org.au/wp/wp-content/uploads/2018/11/PsychologyWeek-2018-Australian-Loneliness-Report.pdf

8. Qualter, P., Vanhalst, J., Harris, R., Van Roekel, E., Lodder, G., Bangee, M., . . Verhagen, M. (2015). Loneliness across the life span. Perspectives on Psychological Science, 10, 250-264. doi:10.1177/1745691615568999

9. Victor, C. R., \& Yang, K. (2012). The prevalence of loneliness among adults: A case study of the United Kingdom. The Journal of Psychology, 146, 85-104. doi:10.1080/00223980.2011.613875

10. Russell, D. W. (1996). UCLA Loneliness Scale (Version 3): Reliability, validity, and factor structure. Journal of Personality Assessment, 66, 20-40. doi:10.1207/s15327752jpa6601_2

11. Cacioppo, S., Grippo, A. J., London, S., Goossens, L., \& Cacioppo, J. T. (2015). Loneliness: Clinical import and interventions. Perspectives on Psychological Science, 10, 238-249. doi:10.1177/1745691615570616

12. Brissette, I., Cohen, S., \& Seeman, T. E. (2000). Measuring social integration and social networks. In S. Cohen, L. G. Underwood, \& B. H. Gottlieb (Eds.), Social support measurement and intervention: $A$ guide for health and social scientists (pp. 53-85). New York, NY, US: Oxford University Press.

13. Reinhardt, J. P., Boerner, K., \& Horowitz, A. (2006). Good to have but not to use: Differential impact of perceived and received support on well-being. Journal of Social and Personal Relationships, 23, 117129. doi:10.1177/0265407506060182

14. Eime, R. M., Young, J. A., Harvey, J. T., Charity, M. J., \& Payne, W. R. (2013). A systematic review of the psychological and social benefits of participation in sport for children and adolescents: Informing development of a conceptual model of health through sport. International Journal of Behavioral Nutrition and Physical Activity, 10. doi:10.1186/1479-5868-10-98

15. Madge, C., Meek, J., Wellens, J., \& Hooley, T. (2009). Facebook, social integration and informal learning at university: 'It is more for socialising and talking to friends about work than for actually doing work'. Learning, Media and Technology, 34, 141-155. doi:10.1080/17439880902923606

16. Shepherd, H., \& Lane, J. (2019). In the mix: Social integration and social media adoption. Social Science Research, 82, 1-17. doi:10.1016/j.ssresearch.2019.02.004 
17. Kingery, J. N., \& Erdley, C. A. (2007). Peer experiences as predictors of adjustment across the middle school transition. Education \& Treatment of Children, 30, 73-88. doi:10.1353/etc.2007.0007

18. Matthews, T., Danese, A., Wertz, J., Odgers, C. L., Ambler, A., Moffitt, T. E., \& Arseneault, L. (2016). Social isolation, loneliness and depression in young adulthood: A behavioural genetic analysis. Social Psychiatry Psychiatric Epidemiology, 51, 339-348. doi:10.1007/s00127-016-1178-7

19. Cacioppo, J. T., Hawkley, L. C., \& Thisted, R. A. (2010). Perceived social isolation makes me sad: 5-year cross-lagged analyses of loneliness and depressive symptomatology in the Chicago Health, Aging, and Social Relations Study. Psychology and Aging, 25, 453-463. doi:10.1037/a0017216

20. Meltzer, H., Bebbington, P., Dennis, M. S., Jenkins, R., McManus, S., \& Brugha, T. S. (2013). Feelings of loneliness among adults with mental disorder. Social Psychiatry and Psychiatric Epidemiology, 48, 513. doi:10.1007/s00127-012-0515-8

21. Steptoe, A., Owen, N., Kunz-Ebrecht, S. R., \& Brydon, L. (2004). Loneliness and neuroendocrine, cardiovascular, and inflammatory stress responses in middle-aged men and women. Psychoneuroendocrinology, 29(5), 593-611. doi:10.1016/s0306-4530(03)00086-6

22. Holt-Lunstad, J., Smith, T. B., Baker, M., Harris, T., \& Stephenson, D. (2015). Loneliness and social isolation as risk factors for mortality: a meta-analytic review. Perspective on Psychological Sciences, 10, 227-237. doi:10.1177/1745691614568352

23. Beran, T. N., Rinaldi, C., Bickham, D. S., \& Rich, M. (2012). Evidence for the need to support adolescents dealing with harassment and cyber-harassment: Prevalence, progression, and impact. School Psychology International, 33, 562-576. doi:10.1177/0143034312446976

24. Guerra, N. G., Williams, K. R., \& Sadek, S. (2011). Understanding bullying and victimization during childhood and adolescence: a mixed methods study. Child Development, 82, 295-310. doi:10.1111/j.1467-8624.2010.01556.x

25. Lawrence, D., Hafekost, J., Johnson, S. E., Saw, S., Buckingham, W. J., Sawyer, M. G., . . Zubrick, S. R. (2015). Key findings from the second Australian Child and Adolescent Survey of Mental Health and Wellbeing. Australian \& New Zealand Journal of Psychiatry, 50, 876-886. doi:10.1177/0004867415617836

26. Laursen, B., \& Hartl, A. C. (2013). Understanding loneliness during adolescence: Developmental changes that increase the risk of perceived social isolation. Journal of Adolescence, 36, 1261-1268. doi:https://doi.org/10.1016/j.adolescence.2013.06.003

27. Qualter, P., Brown, S. L., Rotenberg, K. J., Vanhalst, J., Harris, R. A., Goossens, L., . . Munn, P. (2013). Trajectories of loneliness during childhood and adolescence: predictors and health outcomes. Journal of Adolescence, 36, 1283-1293. doi:10.1016/j.adolescence.2013.01.005

28. Goosby, B. J., Bellatorre, A., Walsemann, K. M., \& Cheadle, J. E. (2013). Adolescent loneliness and health in early adulthood. Sociological Inquiry, 83. doi:10.1111/soin.12018

29. Richard, A., Rohrmann, S., Vandeleur, C. L., Schmid, M., Barth, J., \& Eichholzer, M. (2017). Loneliness is adversely associated with physical and mental health and lifestyle factors: Results from a Swiss national survey. Public Library of Science One, 12, e0181442. doi:10.1371/journal.pone.0181442

30. Lyyra, N., Valimaa, R., \& Tynjala, J. (2018). Loneliness and subjective health complaints among schoolaged children. Scandinavian Journal of Public Health, 46, 87-93. doi.org:10.1177/1403494817743901

31. Nicolaisen, M., \& Thorsen, K. (2014). Who are lonely? Loneliness in different age groups (18-81 Years Old), using two measures of loneliness. The International Journal of Aging and Human Development, 78, 229-257. doi:10.2190/AG.78.3.b

32. Pyle, E., \& Evans, D. (2018). Loneliness-What characteristics and circumstances are associated with feeling lonely? Retieved from https://www.ons.gov.uk/peoplepopulationandcommunity/wellbeing/articles/lonelinesswhatcharacte risticsandcircumstancesareassociated withfeelinglonely/2018-04-10 
33. Rönkä, A. R., Rautio, A., Koiranen, M., Sunnari, V., \& Taanila, A. (2013). Experience of loneliness among adolescent girls and boys: Northern Finland Birth Cohort 1986 study. Journal of Youth Studies, 17, 183-203. doi:10.1080/13676261.2013.805876

34. Yang, K., \& Victor, C. (2011). Age and loneliness in 25 European nations. Ageing and Society, 31, 13681388. doi:10.1017/s0144686x1000139x

35. Hawkins-Elder, H., Milfront, T. L., Hammond, M. D., \& Sibley, C. G. (2018). Who are the lonely? A typology of loneliness in New Zealand. Australian \& New Zealand Journal of Psychiatry, 52, 357-364. doi: $10.1177 / 0004867417718944$

36. Luhmann, M., \& Hawkley, L. C. (2016). Age differences in loneliness from late adolescence to oldest old age. Develomental Psychology, 52, 943-959. doi:10.1037/dev0000117

37. Matthews, T., Danese, A., Caspi, A., Fisher, H. L., Goldman-Mellor, S., Kepa, A., . . Arseneault, L. (2018). Lonely young adults in modern Britain: Findings from an epidemiological cohort study. Psychological Medicine, 49, 268-277. doi: 10.1017/S0033291718000788

38. Mullen, R. A., Tong, S., Sabo, R. T., Liaw, W. R., Marshall, J., Nease, D. E., Jr., . . Frey, J. J., 3rd. (2019). Loneliness in primary care patients: A prevalence study. The Annals of Family Medicine, 17, 108-115. doi:10.1370/afm.2358

39. Bagwell, C. L. (2005). Friendship quality and perceived relationship changes predict psychosocial adjustment in early adulthood. Journal of Social and Personal Relationships, 22. doi:10.1177/0265407505050945

40. Lee, C.-Y. S., \& Goldstein, S. E. (2016). Loneliness, stress, and social support in young adulthood: Does the source of support matter? Journal of Youth and Adolescence, 45, 568-580. doi:10.1007/s10964015-0395-9

41. Carmichael, C. L., Reis, H. T., \& Duberstein, P. R. (2015). In your 20s it's quantity, in your 30s it's quality: The prognostic value of social activity across 30 years of adulthood. Psychology and Aging, 30 95-105. doi:10.1037/pag0000014

42. Borys, S., \& Perlman, D. (1985). Gender differences in loneliness. Personality and Social Psychology Bulletin, 11, 63-74. doi:10.1177/0146167285111006

43. Lasgaard, M., Friis, K., \& Shevlin, M. (2016). "Where are all the lonely people?” A population-based study of high-risk groups across the life span. Social Psychiatry and Psychiatric Epidemiology, 51, 1373-1384. doi:10.1007/s00127-016-1279-3

44. Rich Madsen, K., Trab Damsgaard, M., Smith Jervelund, S., Christensen, U., Stevens, G. G. W. J. M., Walsh, S., ... Holstein, B. E. (2016). Loneliness, immigration background and self-identified ethnicity: a nationally representative study of adolescents in Denmark. Journal of Ethnic and Migration Studies, 42, 1977-1995. doi:10.1080/1369183x.2015.1137754

45. Priest, N., Perry, R., Ferdinand, A., Paradies, Y., \& Kelaher, M. (2014). Experiences of racism, racial/ethnic attitudes, motivated fairness and mental health outcomes among primary and secondary school students. Journal of Youth Adolescence, 43, 1672-1687. doi: 10.1007/s10964-0140140-9.

46. Cohen, P., Kasen, S., Chen, H., Hartmark, C., \& Gordon, K. (2003). Variations in patterns of developmental transmissions in the emerging adulthood period. Developmental Psychology, 39, 657669. doi:10.1037/0012-1649.39.4.657

47. Levitt, M. J., Silver, M. E., \& Santos, J. D. (2008). Adolescents in transition to adulthood: Parental support, relationship satisfaction, and post-transition adjustment. Journal of Adult Development, 14, 53-63. doi:10.1007/s10804-007-9032-5

48. Cacioppo, J. T., \& Hawkley, L. C. (2009). Perceived social isolation and cognition. Trends in Cognitive Sciences, 13, 447-454. doi.org/10.1016/j.tics.2009.06.005

49. Vanhalst, J., Luyckx, K., Van Petegem, S., \& Soenens, B. (2018). The detrimental effects of adolescents' chronic loneliness on motivation and emotion regulation in social situations. Journal of Youth Adolescence, 47, 162-176. doi:10.1007/s10964-017-0686-4 
50. Ekkekakis, P. (2008). The measurement of affect, mood, and emotion. In G. Tenenbaum, R. Eklund, \& A. Kamata (Eds.), Measurement in sport and exercise psychology (pp. 321-332). Champaign, IL, US: Human Kinetics.

51. Watson, D., \& Clark, L. A. (1988). Development and validation of brief measures of positive and negative affect: The PANAS Scales. Journal of Personality and Social Psychology, 54, 1063-1070. doi:10.1037/0022-3514.54.6.1063

52. Baytemir, K., \& Yildiz, M. A. (2017). Multiple mediation of loneliness and negative emotions in the relationship between adolescents' social anxiety and depressive symptoms. Annals of Psychology, 33, 612-620. dx.doi.org/10.6018/analesps.33.3.269211

53. van Roekel, E., Goossens, L., Verhagen, M., Wouters, S., Engels, R. C., \& Scholte, R. H. (2014). Loneliness, affect, and adolescents' appraisals of company: An experience sampling method study. Journal of Research on Adolescence, 24, 350-363. doi.org/10.1111/jora.12061

54. Van Roekel, E., Scholte, R. H., Engels, R. C., Goossens, L., \& Verhagen, M. (2015). Loneliness in the daily lives of adolescents: An experience sampling study examining the effects of social contexts. The Journal of Early Adolescence, 35, 905-930. doi.org/10.1177/0272431614547049

55. Stewart, M., Craig, D., MacPherson, K., \& Alexander, S. (2001). Promoting positive affect and diminishing loneliness of widowed seniors through a support intervention. Public Health Nursing, 18, 54-63. doi.org/10.1046/j.1525-1446.2001.00054.x

56. Fredrickson, B. L. (2000). Cultivating positive emotions to optimize health and well-being. Prevention and Treatment, 3, 1. doi:10.1037/1522-3736.3.1.31a

57. Algoe, S. B., Fredrickson, B. L., \& Gable, S. L. (2013). The social functions of the emotion of gratitude via expression. Emotion, 13, 605-609. doi:10.1037/a0032701

58. Keltner, D., \& Kring, A. M. (1998). Emotion, social function, and psychopathology. Review of General Psychology, 2, 320-342. doi:10.1037/1089-2680.2.3.320

59. Reis, H. T., Smith, S. M., Carmichael, C. L., Caprariello, P. A., Tsai, F. F., Rodrigues, A., \& Maniaci, M. R. (2010). Are you happy for me? How sharing positive events with others provides personal and interpersonal benefits. Journal of Personality and Social Psychology, 99, 311-329. doi:10.1037/a0018344

60. Kearns, S. M., \& Creaven, A.-M. (2017). Individual differences in positive and negative emotion regulation: Which strategies explain variability in loneliness? Personality and Mental Health, 11, 6474. doi:10.1002/pmh.1363

61. Marroquin, B., Czamanski-Cohen, J., Weihs, K. L., \& Stanton, A. L. (2016). Implicit loneliness, emotion regulation, and depressive symptoms in breast cancer survivors. Journal of Behavioral Medicine, 39, 832-844. doi:10.1007/s10865-016-9751-9

62. Marroquin, B., \& Nolen-Hoeksema, S. (2015). Emotion regulation and depressive symptoms: Close relationships as social context and influence. Journal of Personality and Social Psychology, 109, 836855. doi:10.1037/pspi0000034

63. Lubben, J., \& Gironda, M. (2003). Centrality of social ties to the health and well-being of older adults. In B. Berkman \& L. Harootyan (Eds.), Social work and health care in an aging society (pp. 319-350). New York, Springer Publishing Company Inc.

64. Australian Bureau of Statistics. (2018). Socio-economic indexes for areas. Retrieved 19 August 2019

65. Lubben, J., Blozik, E., Gillmann, G., lliffe, S., von Renteln Kruse, W., Beck, J. C., \& Stuck, A. E. (2006). Performance of an abbreviated version of the Lubben Social Network Scale among three European community-dwelling older adult populations. The Gerontologist, 46, 503-513. doi:10.1093/geront/46.4.503

66. Radloff, L. S. (1977). The CES-D Scale: A self-report depression scale for research in the general population. Applied Psychological Measurement, 1, 385-401. doi:10.1177/014662167700100306 
67. Stockings, E., Degenhardt, L., Lee, Y. Y., Mihalopoulos, C., Liu, A., Hobbs, M., \& Patton, G. (2015). Symptom screening scales for detecting major depressive disorder in children and adolescents: A systematic review and meta-analysis of reliability, validity and diagnostic utility. Journal of Affective Disorders, 174, 447-463. doi:10.1016/j.jad.2014.11.061

68. Vilagut, G., Forero, C. G., Barbaglia, G., \& Alonso, J. (2016). Screening for depression in the general population with the Center for Epidemiologic Studies Depression (CES-D): A systematic review with meta-analysis. Public Library of Science One, 11, e0155431. doi:10.1371/journal.pone.0155431

69. Connor, K. M., Kobak, K. A., Churchill, L. E., Katzelnick, D., \& Davidson, J. R. T. (2001). Mini-Spin: A brief screening assessment for generalized social anxiety disorder. Depression and Anxiety, 14, 137-140. doi:10.1002/da.1055

70. Ranta, K., Kaltiala-Heino, R., Rantanen, P., \& Marttunen, M. (2012). The Mini-Social Phobia Inventory: Psychometric properties in an adolescent general population sample. Comprehensive Psychiatry, 53(5), 630-637. doi:10.1016/j.comppsych.2011.07.007

71. Fogliati, V. J., Terides, M. D., Gandy, M., Staples, L. G., Johnston, L., Karin, E., . . Dear, B. F. (2016). Psychometric Properties of the Mini-Social Phobia Inventory (Mini-SPIN) in a large online treatmentseeking sample. Cognitve Behavioral Therapy, 45, 236-257. doi:10.1080/16506073.2016.1158206

72. Gross, J. J., \& John, O. P. (2003). Individual differences in two emotion regulation processes: Implications for affect, relationships, and well-being. Journal of Personality and Social Psychology, 85, 348-362. doi:10.1037/0022-3514.85.2.348

73. Gullone, E., \& Taffe, J. (2012). The Emotion Regulation Questionnaire for Children and Adolescents (ERQ-CA): A psychometric evaluation. Psychological Assessment, 24, 409-417. doi:10.1037/a0025777

74. Lim, M. H., Gleeson, J. F. M., Rodebaugh, T. L., Eres, R., Long, K. M., Casey, K., . . Penn, D. L. (2019). A pilot digital intervention targeting loneliness in young people with psychosis. Social Psychiatry Psychiatric Epidemiology. doi:10.1007/s00127-019-01681-2

75. Lim, M. H., Penn, D. L., Thomas, N., \& Gleeson, J. F. M. (2019). Is loneliness a feasible treatment target in psychosis? Social Psychiatry and Psychiatric Epidemiology. doi:10.1007/s00127-019-01731-9

76. Bruce, L. D., Wu, J. S., Lustig, S. L., Russell, D. W., \& Nemecek, D. A. (2019). Loneliness in the United States: A 2018 national panel survey of demographic, structural, cognitive, and behavioral characteristics. American Journal of Health Promotion. doi: https://doi.org/10.1177/0890117119856551

77. Shevlin, M., Murphy, S., Mallett, J., Stringer, M., \& Murphy, J. (2013). Adolescent loneliness and psychiatric Morbidity in Northern Ireland. British Journal of Clinical Psychology, 52, 230-234. doi:10.1111/bjc.12018

78. Miething, A., Almquist, Y. B., Ostberg, V., Rostila, M., Edling, C., \& Rydgren, J. (2016). Friendship networks and psychological well-being from late adolescence to young adulthood: A gender-specific structural equation modeling approach. BioMed Central Psychology, 4, 34. doi:10.1186/s40359-0160143-2

79. Dagnew, B., \& Dagne, H. (2019). Year of study as predictor of loneliness among students of University of Gondar. BMC Res Notes, 12, 240. doi:10.1186/s13104-019-4274-4

80. Diehl, K., Jansen, C., Ishchanova, K., \& Hilger-Kolb, J. (2018). Loneliness at universities: Determinants of emotional and social loneliness among students. International Journal of Environmental Research and Public Health, 15. doi:10.3390/ijerph15091865

81. Eisenberg, D., Gollust, S. E., Golberstein, E., \& Hefner, J. L. (2007). Prevalence and correlates of depression, anxiety, and suicidality among university students. American Journal of Orthopsychiatry, 77, 534-542. doi:10.1037/0002-9432.77.4.534

82. Holt-Lunstad, J., Smith, T. B., \& Layton, J. B. (2010). Social Relationships and Mortality Risk: A Metaanalytic Review. Pulic Library of Science Medicine, 7, e1000316. doi:10.1371/journal.pmed.1000316

83. Adam, E. K., Chyu, L., Hoyt, L. T., Doane, L. D., Boisjoly, J., Duncan, G. J., . . McDade, T. W. (2011). Adverse adolescent relationship histories and young adult health: Cumulative effects of loneliness, 
low parental support, relationship instability, intimate partner violence, and loss. Journal of Adolescent Health, 49, 278-286. doi:10.1016/j.jadohealth.2010.12.012

84. Van Gelder, M. M., Bretveld, R. W., \& Roeleveld, N. (2010). Web-based questionnaires: the future in epidemiology? American Journal of Epidemiology, 172, 1292-1298. doi:10.1093/aje/kwq291

85. Mattick, R. P., \& Clarke, J. C. (1998). Development and validation of measures of social phobia scrutiny fear and social interaction anxiety. Behaviour Research and Therapy, 36, 455-470. doi:10.1016/S00057967(97)10031-6

86. Danneel, S., Bijttebier, P., Bastin, M., Colpin, H., Van den Noortgate, W., Van Leeuwen, K., . . Goossens, L. (2019). Loneliness, social anxiety, and depressive symptoms in adolescence: Examining their distinctiveness through factor analysis. Journal of Child and Family Studies, 28, 1326-1336. doi:10.1007/s10826-019-01354-3

87. Maes, M., Nelemans, S. A., Danneel, S., Fernandez-Castilla, B., Van den Noortgate, W., Goossens, L., \& Vanhalst, J. (2019). Loneliness and social anxiety across childhood and adolescence: Multilevel metaanalyses of cross-sectional and longitudinal associations. Developmental Psychology, 55, 1548-1565. doi:10.1037/dev0000719

88. Lim, M. H., Rodebaugh, T. L., Eres, R., Long, K. M., Penn, D. L., \& Gleeson, J. F. M. (2019). A pilot digital intervention targeting loneliness in youth mental health. Frontiers in Psychiatry - Mood and Anxiety Disorders. doi.org/10.3389/fpsyt.2019.00604

89. Donetto, S., Pierri, P., Tsianakas, V., \& Robert, G. (2015). Experience-based co-design and healthcare improvement: Realizing participatory design in the public sector. The Design Journal, 18, 227-248. doi:10.2752/175630615X14212498964312

90. Ennis, L., \& Wykes, T. (2013). Impact of patient involvement in mental health research: longitudinal study. British Journal of Psychiatry, 203, 381-386. doi:10.1192/bjp.bp.112.119818 


\section{VicHealth}

Victorian Health Promotion Foundation PO Box 154 Carlton South

Victoria 3053 Australia

$T+61396671333 F+61396671375$

vichealth@vichealth.vic.gov.au

vichealth.vic.gov.au

twitter.com/vichealth

facebook.com/vichealth

VicHealth acknowledges the support of the Victorian Government.

(C) VicHealth 2019

September 2019 P-MW-811

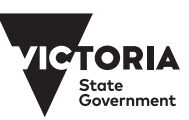

